Title:

Eelectrochemical Deposition of Silver and Copper from a Deep Eutectic Solvent Studied Using Time-Resolved Neutron Reflectivity.

Graphical Abstract:

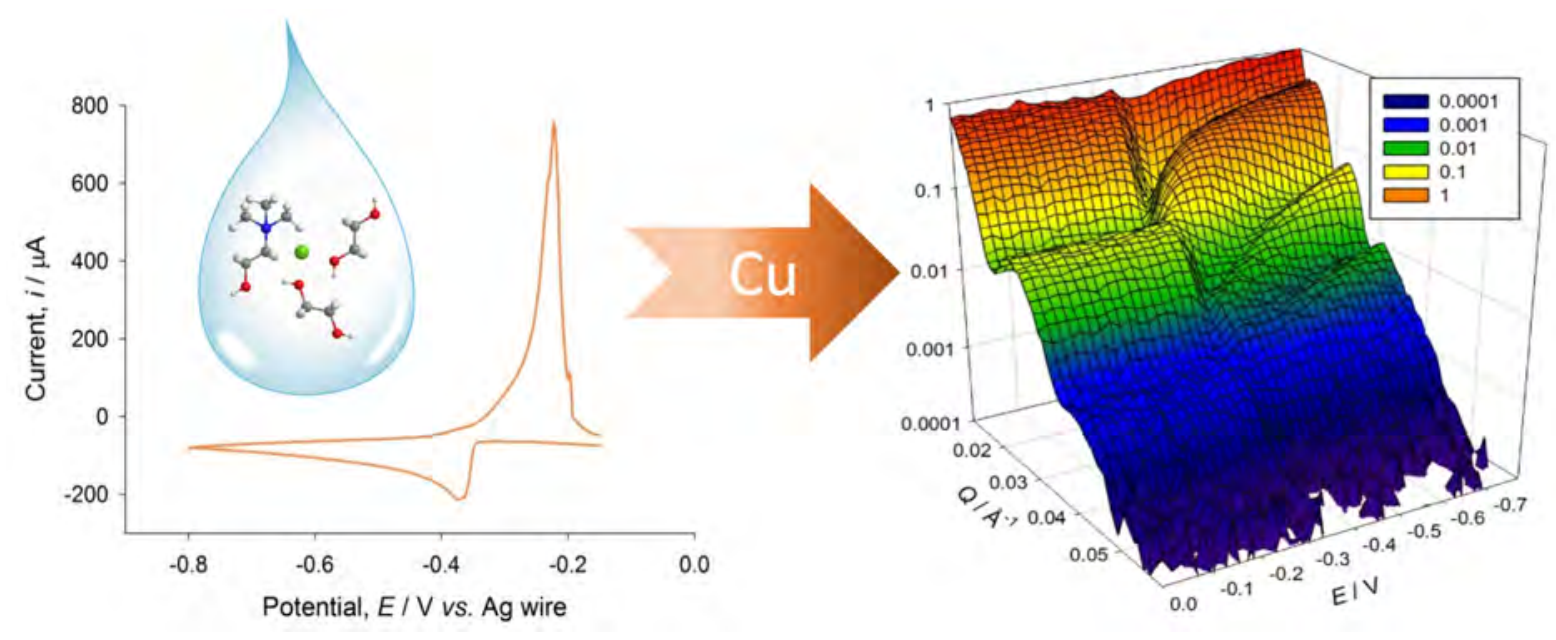

Research Highlights:

- Real-time analysis of electrodeposited metal films using neutron reflectivity methods

- Thickness, roughness and porosity of films analyzed during electrochemical growth

- Electrochemical control function influences properties of growing metal film

- Copper shows a stronger dependency on deposition conditions than does silver

- Robust and stable approach to the fitting of NR data demonstrated 


\title{
Electrochemical Deposition of Silver and Copper from a Deep Eutectic Solvent Studied Using Time-Resolved Neutron Reflectivity.
}

\author{
Andrew D. Ballantyne ${ }^{1}$, Robert Barker ${ }^{2}$, Robert M. Dalgliesh ${ }^{3}$, \\ Virginia C. Ferreira ${ }^{4}$, A. Robert Hillman ${ }^{1}$, Emma J. R. Palin ${ }^{1}$, Rachel Sapstead ${ }^{1}$, \\ Emma L. Smith ${ }^{5}$, Nina-Juliane Steinke ${ }^{3}$ and Karl S. Ryder ${ }^{{ }^{*}}$ \\ *k.s.ryder@le.ac.uk \\ 1 Materials Centre, Department of \\ Chemistry, University of Leicester, \\ University Road, Leicester LE1 7RH, UK. \\ 3 Rutherford Appleton Lab, ISIS Facility, \\ STFC, Harwell, OX11 0QX, UK. \\ Department of Chemistry, School of \\ Science and Technology, Nottingham \\ Trent University, Nottingham NG11 8NS, \\ UK.
}

\section{Abstract}

Here, we describe new developments in the study of electrodeposition processes with timeresolved dynamic neutron reflectivity (NR) methods to achieve insights into the differences between growth of metal films using a range of electrochemical control functions. We show that the temporal resolution of the here has increased from 1-2 hours per data set (in our previous studies) to approximately 8 minutes. We have studied the electrochemical deposition of copper and silver as thin-film metals onto a gold electrode substrate from a deep eutectic solvent using potentiodynamic (PD), potentiostatic (PS) and galvanostatic (GS) electrochemical control functions. In particular, we have utilised novel developments in neutron reflectivity methods to acquire real-time data for the growing metal films. Event mode capture of neutron scattering events, as a function of momentum transfer vector, $Q$, during electrochemical growth has enabled time-resolved measurement of the neutron reflectivity, $R(Q)$, profiles of the growing metal films. Subsequent fitting and iterative optimisation of the $R(Q, t)$ data reveals the thickness, roughness and relative density (spatially resolved solvent content) of the metal film during growth. These data show that the different electrochemical growth methodologies exhibit different trends in thickness, roughness and solvation. Silver films show an increasing roughness trend with time but these trends are largely independent of growth method. In contrast, the roughness of copper films, grown under similar conditions, shows a strong dependency on growth method with PS methods producing smoothest films. These conclusions are confirmed by ex-situ AFM measurements. The fitted NR data show that the $\mathrm{Cu}$ and $\mathrm{Ag}$ films contain between $5-10 \%$ volume fraction solvent. Furthermore, we have explored different NR data fitting methodologies in order to process the large numbers of data sets produced. Gratifyingly, the different methodologies and starting conditions yield a very consistent picture of metal film growth.

Keywords: Neutron reflectivity, event mode, electrodeposition, deep eutectic solvents, copper, silver, thin-film, cyclic voltammetry, atomic force microscopy. 


\section{Introduction}

Electrodeposition of metal films and coatings is a core practice for a variety of high technology and manufacturing industries. The range of metals of interest is very wide and includes $\mathrm{Cr}$, $\mathrm{Ni}, \mathrm{Cd}, \mathrm{Cu}, \mathrm{Zn}$ and $\mathrm{Sn}$, for anti-corrosion and wear coatings used in automotive, aerospace and many other industries. $\mathrm{Au}, \mathrm{Ag}$ and $\mathrm{Pt}$, are important for electronics fabrication and manufacturing and $\mathrm{Al}, \mathrm{Li}, \mathrm{Na}, \mathrm{Co}$ and $\mathrm{Mn}$ for energy storage applications, including novel batteries. In many applications, it is necessary to control the physical properties, morphology, roughness, and density of the metal film produced by electro-reduction of metal ion. This is particularly the case where thin films are required. Such technologies include electronics fabrication and manufacturing, including printed circuit boards (PCB). In the latter case multilayer thin films of, for example, $\mathrm{Ni}, \mathrm{Cu}, \mathrm{Ag}, \mathrm{Au}$ and others are often produced by electrolytic reduction.

The overwhelming majority of electrolytic metal processing is carried out in water based solutions. However, despite their maturity, aqueous deposition processes still possess significant limitations such as low current efficiency, coating embrittlement and dendrite formation; stringent process control is often necessary to maintain specification which leads to bath complexity and rigorous maintenance requirements. ${ }^{1}$ In addition, strong inorganic acids and bases are often needed and the metal salts required, for example cyanides, are often very toxic. Consequently, the use of novel ionic liquid ${ }^{2}$ (IL) media and in particular deep eutectic solvents ${ }^{3}$ (DES) is gaining attention. DESs are systems formed from eutectic mixtures of Brønsted or Lewis acids and bases, typically mixtures of the salt choline chloride with small hydrogen bonding molecules such as ethylene glycol or urea. ${ }^{3}$ DES based electrolytes have been used for the electrodeposition of a wide range of metal and alloy coatings including $\mathrm{Cu}$, ${ }^{4,5} \mathrm{Sn},{ }^{6,7,8} \mathrm{Zn},{ }^{9,10} \mathrm{Cr},{ }^{11,12} \mathrm{Zn} / \mathrm{Ni}^{13}$ and $\mathrm{Zn} / \mathrm{Sn} .{ }^{14}$ These DES electrolyte media offer prospective improvements in process control/efficiency, environmental sustainability/impact and functionality as well as giving access to reactive metal deposition not previously possible (for example Al). In addition, due to the fundamental differences between molecular solvents like water and ILS/DESs there are significant disparities in the way metal films nucleate and grow on surfaces. ${ }^{15}$ 
Electrochemical reduction is driven by control of either the applied potential, $E_{A p p} / \mathrm{V}$, or the current density, $i / \mathrm{A} \mathrm{cm}^{-2}$. Since one is a function of the other there are two generic methodologies for deposition of a metal film; these are potential control and current control. Potential control is achieved most simply by applying a fixed value of cathodic potential sufficient to drive the reduction process. In this case the resultant current density varies with time, $i(t)$, according to the availability of metal ion species and/or the rate of limiting kinetic factors (heterogeneous electron-transfer, metal complex ligand exchange etc.). This is the potentiostatic method (PS) and here the thermodynamic driving force, $E_{A p p .,}$ is constant but the rate of reaction, $i$, is (in general) a function of time. Alternatively, current control is achieved by driving a fixed value of current through the cell in the direction of the reduction reaction. In this case the value of applied potential required to sustain the chosen current will vary with time for the same reasons (above). This is the galvanostatic method (GS) and here the rate of reaction, $i$, is fixed but the driving force, $E_{A p p}$, is a function of time.

During PS deposition, the rates of nucleation and growth of the metal film are determined by the fixed overpotential. This often results in control over the nucleation mechanism and possibly grain size/structure but can lead to very slow deposition rates at long experimental time scales. In contrast, GS methods offer control of reduction rate but this can lead to large variations in applied potential and current efficiency which, in turn, can cause roughness due to the variation in nucleation and growth kinetics. A third option is to gradually sweep the applied potential in the cathodic direction from a starting, quiescent, potential through the kinetically controlled region of the voltammetric reduction peak to a final value in the diffusion controlled region. This is a potentiodynamic (PD) (linear sweep voltammetry) method. Sweeping the potential in this way offers control of the initial nucleation conditions, at low rate, but then increases the rate of deposition at longer times through gradual, but controlled, increases of the applied potential. In this way the PD method combines some of the attractive features of both the PS and GS methods.

Each of these three methods of electrolytic growth can produce coatings with different physical attributes because of the limiting conditions under which growth is maintained. Consequently, a detailed understanding of how these control functions influence the structure and properties of the growing metal film is critical to being able to reproduce consistent metal films to a prescribed thickness and specification. 
We have been engaged in the study of electrolytic metal deposition and dissolution in DES media in relation to potential applications in the aerospace and electronics manufacturing sectors. ${ }^{16}$ Here it is important to be able to control and predict rate of deposition of the metal as well as to achieve target values of surface roughness and coherent, dense coatings. Monitoring thickness, surface roughness and density during electrodeposition can be achieved by a combination of techniques including electro-gravimetry (Quartz Crystal Microbalance, QCM) ${ }^{17}$, holographic imaging (Digital Holographic Microscopy, DHM) ${ }^{18}$, scanning electron microscopy (SEM), optical profiling ${ }^{19}$ and scanning probe microscopy (Atomic Force Microscopy, AFM) ${ }^{20}$ as well as integrated electrochemical techniques (chronocoulometry). Each of these techniques has individual strengths but cannot in isolation deliver the necessary insights and metrology. Electrochemical and QCM measurements have good temporal resolution but are averaged over the sample volume (both across the surface and throughout its depth). Optical microscopy is limited by line of sight access to the electrode surface and the spatial resolution of visible wavelengths, whereas high resolution techniques such as SEM can only be utilised ex-situ. Probe microscopy can offer great insight into the shape of an evolving surface during growth ${ }^{21}$ and into the mechanism of growth, but the proximity of the sharp probe close to the electroactive interface, or touching it, can initiate nucleation events and thus perturb the measurement. Furthermore, none of these techniques is capable of quantifying the internal composition of the deposited film during deposition.

An alternative is to use neutron reflectivity, NR, techniques. ${ }^{22}$ This approach has many similarities to optical ellipsometry although the metal coatings here are optically opaque and so ellipsometry is not appropriate. Neutron reflectivity techniques are able to provide not only thickness and roughness data for the growing film but also compositional detail perpendicular to the plane of the electrode i.e. in the direction of growth. Development of NR methods to study "buried" interfaces under electrochemical control has distinguished composite and bilayer polymer films, ${ }^{23}$ revealed permeating solvent in electroactive polymer ${ }^{24}$ and metal hydroxide ${ }^{25}$ films, identified permselectivity failure at high electrolyte concentration, ${ }^{26}$ and revealed 1D profiling of diffusion and reaction within a film of a solution phase mediator. ${ }^{27}$ Of relevance here, we have used a combination of in-situ techniques including NR to evaluate the influence of electrolytic deposition conditions on the solvation 
of reactive conducting polymers. ${ }^{28,29}$ These and related ${ }^{30,31}$ NR studies have data acquisition time scales of 1-2 hours per acquisition, so the equilibrated films can be observed, as a function of potential and/or charge, but not the dynamics of interconversion.

In previous studies, we have been able to achieve better effective temporal resolution by signal averaging over repetitive scans and have reported the first dynamic electrochemical NR measurements by repetitive scan voltammetry. ${ }^{32}$ This enabled the study of the time and potential dependent thickness and solvation of a poly(vinyl ferrocene) film. However, that strategy is inappropriate for 'single-shot' experiments. More recently, developments in instrument technology and data processing at the ISIS neutron and muon source, Rutherford Appleton Laboratory, have resulted in greatly improved temporal resolution. Data capture in event mode allows monitoring and recording of individual neutron events. This enables the combination (statistical averaging) of neutron data, post-acquisition, over a flexible, userdefined time scale. The subsequent time scale required for a single $R(Q)$ profile can be as little as tens of seconds in principle (cf. 1-2 hours earlier), however, the cost of increasing temporal resolution is increased signal:noise (in $R$ and $Q$ ). A crucial advantage of the event mode experiment is that it offers the capability to process all the acquired data after the experiment (without introducing dead time during data acquisition). This provides flexibility in achieving the desired balance of temporal resolution and noise and removes ambiguities associated with sample relaxation during interrupted measurements. We have recently exploited these new developments to study the electrochemical NR dynamics of both metal deposition and conducting polymer growth. ${ }^{33}$

Here we have achieved good signal to noise in the NR data at temporal resolution of typically 5-10 minutes but varying to suit experimental needs. Event mode capture of synchronous neutron scattering events during electrochemical growth has enabled time-resolved measurement of the neutron reflectivity profiles of the growing metal films.

In this study, we apply these developments in electrochemical NR measurements to investigate silver and copper metal growth in a deep eutectic solvent medium using three different electrochemical control functions: potentiostatic, galvanostatic and potentiodynamic. Specifically, our objectives have been to use these methods to determine the thickness, roughness and compositional uniformity (porosity, solvent content) of the growing metal film, to make quantitative comparisons between the two metals and to 
determine the effect on these parameters of the electrochemical growth control regime. The issue of metal deposit composition (e.g. chloride or hydrogen content) and porosity is an important one for applications of metal coatings because it directly influences performance criteria such as mechanical integrity/strength and corrosion/wear resistance. In a separate study, we are using NR techniques, including H/D isotopic substitution, to measure hydrogen content and embrittlement of films deposited in DES media. The outcomes of that study will be published elsewhere. Furthermore, we have set out to build confidence in the outputs of the numerical fitting and data processing methods for this type of experimental NR data and to optimise the process for large numbers of data sets. To achieve this, we have processed multiple data sets using a variety of initial conditions for the film model with two separate software packages (RasCal and MotoFit) utilising both parallel and serial methodologies. ${ }^{34,35}$

\section{Experimental}

\section{Reagents and Materials}

All chemicals were used as supplied. Silver chloride and copper (II) chloride were supplied by Acros Organics. Choline chloride $(\mathrm{ChCl})$, ethylene glycol (EG) and 3-mercaptopropyl trimethoxysilane (MPTS) were supplied by Sigma Aldrich.

Ethaline 200 was synthesised from a 2:1 molar ratio of ethylene glycol $\left(62.07 \mathrm{~g} \mathrm{~mol}^{-1}\right)$ and choline chloride $\left(139.62 \mathrm{~g} \mathrm{~mol}^{-1}\right)$. The mixture was stirred at $70^{\circ} \mathrm{C}$ until a clear, homogeneous solution was observed. Metal chloride solutions were prepared to a $10 \mathrm{mM}$ concentration in Ethaline 200 by stirring at $50^{\circ} \mathrm{C}$ until dissolved.

For NR experiments, the Au coated quartz working electrode was prepared by first coating the quartz with a monolayer of 3-mercaptopropyl trimethoxysilane (MPTS). ${ }^{26} \mathrm{~A}$ binding layer was used to ensure that the Au did not delaminate from the quartz by exploiting the Au-S affinity. Following this, a gold layer was sputter coated onto the MPTS binding layer to a thickness of $c a$. 20-30 $\mathrm{nm}$. In the case of all experiments, a platinum mesh was used as a counter electrode, and Ag wire as a quasi reference electrode. These were assembled in a standard three electrode cell configuration inside a purpose built electrochemical cell. ${ }^{27,28}$ 


\section{Instrumentation}

NR measurements were performed at room temperature on OFFSPEC at the ISIS neutron and muon source at the Rutherford Appleton Laboratory (Harwell, Oxford, UK.) All measurements were carried out in event mode. Data were 'time sliced' into $500 \mathrm{~s}$ sections postmeasurement and exported to the fitting software (see below). An incident angle $(\theta)$ of $0.5^{\circ}$ was used, allowing for a $\lambda$ range of 1.0 - $14 \AA$, and a useable momentum transfer, $Q$ (see NR methods), range of $0.008<Q / \AA^{-1}<0.07$. The neutron beam footprint was constrained to within the cell dimensions giving an effective instrumental resolution of $\Delta Q / Q \sim 2 \%$. The cell volume was $25 \mathrm{~cm}^{3}$. The start of the electrochemical reaction was triggered by the neutron instrument and simultaneously recorded in the neutron data file so that the neutron data acquisition times accurately match the electrochemical experiment times. All electrochemical experiments were carried out using an IVIUM CompactStat potentiostat controlled by IviumSoft software version 2.224 .

The instrument used for AFM measurements was a Veeco Dimension 3100 Scanning Probe Microscope with Veeco Silicon Probes. Images were recorded in tapping mode, and analysed using Nanoscope (version 6.12rl) software. Roughness values were taken as ' $R_{q}$ ', which is the root mean squared value.

\section{Electrochemical Procedures}

\section{Potentiostatic Measurements}

Copper (from $10 \mathrm{mM} \mathrm{CuCl}_{2}$ ) and silver (from $10 \mathrm{mM} \mathrm{AgCl}$ ) in Ethaline 200 were deposited at a constant potential vs. Ag wire onto the Au coated quartz working electrode, with an area of ca $28 \mathrm{~cm}^{2}$. Cu was deposited at $-0.6 \mathrm{~V}$ for $18.1 \times 10^{3} \mathrm{~s}(5 \mathrm{hrs})$ and silver at $-0.2 \mathrm{~V}$ for $14.4 \times 10^{3}$ s (4 hrs.) The resulting $i$ vs. $t$ traces were recorded. During the electrochemical depositions in the NR cell, less than $10 \%$ of the metal ion salt in solution was consumed in each case. The electrochemical cell for NR measurements was assembled using Dow Corning (3145 RTV-Clear MIL-A-46146) adhesive sealant.

\section{Potentiodynamic Measurements}

Copper and silver (as above) were deposited over a single cathodic linear sweep at a scan rate of $0.02 \mathrm{mV} \mathrm{s}^{-1}$. In the case of copper, the potential was swept between $0 \mathrm{~V}$ and $-0.8 \mathrm{~V}(40$ 
$\left.\mathrm{x} 10^{3} \mathrm{~s}\right)$ and silver between $0.2 \mathrm{~V}$ and $-0.3 \mathrm{~V}\left(25 \times 10^{3} \mathrm{~s}\right)$, and the resulting $i$ vs. $E$ traces were recorded.

\section{Galvanostatic Measurements}

Copper and silver (as above) were deposited at a constant current. In this case, a potential limit of $-1.0 \mathrm{~V}$ was employed and experiments carried out until this was reached. Copper was deposited at a constant current of $-330 \mu \mathrm{A}\left(-11.79 \mu \mathrm{A} \mathrm{cm}{ }^{-2}\right)$ for $5360 \mathrm{~s}(1.48 \mathrm{hrs})$ and silver at a constant current of $-120 \mu \mathrm{A}\left(-4.29 \mu \mathrm{A} \mathrm{cm}{ }^{-2}\right)$ for $12728 \mathrm{~s}(3.5 \mathrm{hrs})$. The resulting $E v s . t$ traces recorded. In each case the experiment was terminated when the applied potential required to drive the chosen current reached $-1.0 \mathrm{~V}$ (the voltage limit, or "cap").

\section{NR Data Analysis}

All data fitting was carried out using either MotoFit ${ }^{34}$ (functioning within Igor) or RasCal ${ }^{35}$ (functioning as a script within Matlab.) Both types of software involve iterative fitting procedures using multi-parameter models. Data fitting errors were determined using a "bootstrap" error analysis function within RasCal. ${ }^{36}$

\section{Results and discussion}

\section{Electrochemistry of Cu and Ag salts in DES:}

Both copper and silver chlorides $\left(\mathrm{CuCl}_{2}\right.$ and $\left.\mathrm{AgCl}\right)$ are soluble in the deep eutectic solvent formulated from ethylene glycol $(\mathrm{Eg})$ and choline chloride $(\mathrm{ChCl})$ in the stoichiometric ratio 2(Eg): $\mathrm{ChCl}$. This solvent is known by its commercial name as Ethaline 200. Ethaline 200 was chosen for this study because it has been shown to be effective in many electrolytic metal deposition/dissolution processes as an alternative to aqueous electrolytes and is relatively inexpensive and easy to prepare. ${ }^{3}$ Each of these metal ions exhibits a well-behaved chemically reversible redox reaction resulting in metal deposition and stripping. The cyclic voltammograms for $\mathrm{CuCl}_{2}$ and $\mathrm{AgCl}$ in Ethaline 200 are shown in Figure 1. Each of these voltammograms shows a diffusion controlled one electron reduction, $M^{+/ 0}(M=C u$ or $A g)$, resulting in metal deposition followed by oxidation and dissolution of deposited metal. The associated phase change during dissolution is evident from the shape and symmetry of the anodic peak. In the case of $\mathrm{Cu}$, the reversible $\mathrm{Cu}^{2+/+}$ couple is also observed at more positive 
potentials (not shown). We have published previously on the electrochemistry of these metal ions in DES. ${ }^{5,37}$ Electrodeposition of either metal by sustained reduction results in the formation of a coherent metal film that is optically smooth and bright. However, as with many kinetic phenomena, the physical properties of the deposited film (morphology, roughness, porosity and density) can be strongly dependent on the conditions imposed. The aim of this study is to identify and characterise those variations.

For the purposes of this study, copper and silver films were deposited on the face of a gold coated single crystal quartz block (see below) under potential control (PS) at a value of applied potential (versus the $\mathrm{Ag}$ wire reference electrode) in the diffusion controlled regime defined by voltammetry, Figure 1 . For silver, this corresponded to a value of $-0.2 \mathrm{~V}$ and for copper, a value of $-0.6 \mathrm{~V}$. The corresponding diffusion-controlled current reached in each case reached a long time limiting value of approximately $-150 \mu \mathrm{A}\left(-5.4 \mu \mathrm{A} \mathrm{cm}{ }^{-2}\right)$ for $\mathrm{Cu}$ and $-100 \mu \mathrm{A}(-3.6 \mu \mathrm{A}$ $\mathrm{cm}^{-2}$ ) for Ag. Under current control (GS) the value of current driven was chosen to correspond to a similar value to the diffusion controlled currents reached in PS growth. These were -330 $\mu \mathrm{A}\left(-11.8 \mu \mathrm{A} \mathrm{cm}^{-2}\right)$ for $\mathrm{Cu}$ and $-120 \mu \mathrm{A}\left(-4.3 \mu \mathrm{A} \mathrm{cm}{ }^{-2}\right)$ for $\mathrm{Ag}$.

The metal films were grown and time-resolved NR data were acquired and monitored during growth. In a typical deposition experiment the film growth was stopped after a period of 4 to 5 hours.

\section{Neutron reflectivity methods:}

The design of the cell used for electrochemical deposition of metal films and synchronous NR data acquisition is shown in Figure 2 . The rectangular electrode surface was a sputter coated gold layer (ca. 20-30 nm) on a single crystal quartz block. The surface of the quartz was pretreated with (3-mercaptopropyl)trimethoxysilane, (MPTS), in order to improve binding of the gold layer (see experimental section). The opposing face of the cell consisted of a glass plate (allowing optical inspection of the interior) over which the counter electrode mesh was secured. The cell was enclosed by a machined PTFE gasket and held together with a stainless steel clamp. The silver wire reference electrode was glued to the side of the PTFE gasket close to the gold working electrode surface. This type of cell design is similar to that which we have used successfully in previous studies. ${ }^{27,28,32,33}$ The incident neutron beam was directed 
through the quartz, to illuminate the quartz/Au interface, i.e. the "dry" side of the electrode/electrolyte interface, Figure 2 (insert). The characteristic neutron reflectivity profiles are a consequence of subsequent interference interactions of the reflected beam at the successive layers of the solid/solid, solid/liquid interfaces. These interfaces can be identified from Figure 2 (insert) and are listed below;

1) quartz/MPTS,

2) MPTS/Au,

3) $\mathrm{Au} / \mathrm{M}$, (where $\mathrm{M}$ represents the growing metal film, $\mathrm{Cu}$ or $\mathrm{Ag}$ ),

4) $M /$ bulk solvent (DES).

The NR data are conventionally presented as the logarithm of Fresnel reflectivity, $R$, (where $R$ is determined by the ratio of the incident and transmitted wave vectors) as a function of the momentum transfer, $Q$, defined in Equation 1.

$$
Q=\frac{4 \pi \sin \theta}{\lambda}
$$

\section{Equation 1}

Here, $Q$, is the magnitude of momentum transfer vector, $\theta$, is the angle of incidence of the neutron beam with the plane of the electrode surface and, $\lambda$, is the De Broglie wavelength of the incident neutron. Thus, for a fixed angle of incidence, $\theta$, the value of $Q$ is a reciprocal function of the wavelength, $\lambda$, of the incident neutron. Consequently, all of the $R(Q)$ data are in reciprocal space. Neutron beams are generated either by a spallation sources (e.g. ISIS, RAL, Oxford, UK) or by research nuclear reactors (e.g. ILL, Grenoble, Fr). The beam used in the experiments here is polychromatic, i.e. it contains a range of neutron energies (wavelengths) and these are resolved by the time of flight (TOF) method. The theory of NR is treated in detail separately elsewhere. ${ }^{22,28}$

Representative NR data sets, $R(Q)$, for a growing $\mathrm{Cu}$ film deposited on the quartz/MPTS/Au substrate are presented as Figure 3a. In this case the $\mathrm{Cu}$ film was grown under PD control by scanning from $0 \mathrm{~V}$ to $-0.8 \mathrm{~V}$ at $0.02 \mathrm{mV} \mathrm{s}^{-1}$ (a total elapsed time of $40 \times 10^{3} \mathrm{~s}$ ). These data were processed into 80 individual $R(Q)$ profiles (only three of which are shown in Figure 3 ) resulting in a temporal resolution of $500 \mathrm{~s}(8.3 \mathrm{~min})$ for each acquisition. Here there are three $R(Q)$ traces, one of the bare Au electrode, $t=0 \mathrm{~s}(0 \mathrm{mV}$ in the voltammogram), one during Cu film growth, $t=28.5 \times 10^{3} \mathrm{~s}$ (corresponding to $570 \mathrm{mV}$ in the voltammogram) and one at the end 
of the experiment after film growth was complete, $t=39.5 \times 10^{3} \mathrm{~s}$ (corresponding to $790 \mathrm{mV}$ in the voltammogram). These data are characterised by the appearance of reflectivity fringes with a periodic spacing, $\Delta Q$. The critical edge is defined as the value of $Q$ at which $R$ is equal to unity although a critical edge is not always observed in the $R(Q)$ profile depending on the identity of the materials comprising the interface. ${ }^{22}$

Here, Figure 3a, the observed fringes are a function of all the interfaces and layers present (see above). However, in the case of a simple, single-layer interface, for example Au on quartz, the thickness of that layer (metal film) can be estimated using Equation 2.

$$
d=\frac{2 \pi}{\Delta Q}
$$

\section{Equation 2}

Here, $d$, represents film thickness of the layer and $\Delta Q$ is the measured spacing (peak to peak) of the relevant reflectivity fringe in the $R(Q)$ profile. In the first of the $R(Q)$ plots, Figure 3a, at $t=0$, there is no copper present, only the Au substrate. In this case the thickness of the Au layer can be estimated by using Equation 2. The value of $\Delta Q$ measured from the profile (0.03$0.02 \AA^{-1}$ ) gives a thickness of approximately 200-300 $\AA$. A more precise value will result from data fitting (see below). As the $\mathrm{Cu}$ deposition progresses, the observed fringe frequency increases, Figure 3a, but the overall effect is a combination and overlap of fringes due to both the Au and Cu layers. Under these circumstances, the thicknesses of the individual layers and components cannot be uniquely determined using Equation 2. In addition, the presence and position (in $Q$ space) of the critical edge and the shape of the reflectivity fringes is determined by the scattering length density and thickness of the adjacent layers. Consequently, in order to extract useful data regarding film thickness, roughness and composition, data-fitting methods must be employed.

The neutron scattering power of a thin-film material is defined as the scattering length density, $S L D$, typically expressed in units of $\AA^{-2}$. This is analogous to an optical refractive index and is unique to material and atom types. For any volume region within a film, the value of $S L D$ is given by the summation, over the elements (isotopes), $i$, present, of the product of their number density, $N_{i}$, (in units of nuclei per $\mathrm{cm}^{3}$ ), and their neutron scattering lengths, $b_{i}$. This is shown in Equation 3. 


$$
S L D=\sum_{i} N_{i} b_{i}=\left(\frac{N_{\mathrm{Av} .} \rho}{\text { r.a.m. }}\right) \sum_{i} n_{i} b_{i}
$$

\section{Equation 3}

Here, $n_{i}$, is the stoichiometry of the element (isotope), $i$, in a material with relative atomic mass, r.a.m., and density, $\rho$, within the medium. $N_{\mathrm{Av}}$ is Avogadro's number. For a single, bulk phase, e.g. a pure metal, the value of SLD can be determined from published data. Importantly, the scattering length density, SLD, of a composite medium, polymer, or solvated film, is a simple additive function of the scattering length densities of its component parts, i.e. atoms.

The data fitting methods take as input a simple physical model that represents an estimate of the interface structures and the measured $R(Q)$ data. The model includes the bulk materials (here quartz and solvent media), the solid/solid, solid/liquid interfaces listed above and estimated layer thickness/roughnesses. The fitting algorithm then minimises the difference between the measured $R(Q)$ profile and a corresponding $R(Q)$ profile calculated using the parameters defined in the model. Importantly here, the output of the fitting algorithm is a profile of the scattering length density, $S L D$, as a function of distance, $z$, perpendicular to the plane of the electrode surface, $\operatorname{SLD}(z)$. For our purposes, this represents a compositional profile where the distance dimension, $z$, corresponds to the direction of growth of a deposited film. The fitting procedures and software tools are described in the experimental section and published in greater detail elsewhere. ${ }^{34,35}$

The fitted scattering length density profiles, $S L D(z)$, for the data, $R(Q)$, in Figure 3a, are shown in Figure $\mathbf{3 b}$. The plane at $z=0$ is defined, arbitrarily, as close to the interface between the bulk quartz $\left(\mathrm{SiO}_{2}\right)$ and the MTPS binding layer. The value of $S L D$ for bulk $\mathrm{SiO}_{2}$ is $4.18 \times 10^{-6} \AA^{-}$ 2. Moving outwards, increasing value of $z$, the scattering length density drops in value corresponding to the presence of the MPTS layer between the quartz and the gold. The value of SLD for bulk MPTS is $0.27 \times 10^{-6} \AA^{-2}$. At values of $z$ in the range $20<z<40 \mathrm{~nm}$ the corresponding values of $S L D$ are constant at approximately $4.5 \times 10^{-6} \AA^{-2}$. This plateau corresponds to the presence of the gold coating on the quartz substrate. Gold metal has a bulk SLD value of $4.49 \times 10^{-6} \AA^{-2}$. Hence the $A u$ is $25 \mathrm{~nm}$ in thickness. This is in good agreement with initial estimates (see above) using Equation 2. The thickness of the MPTS layer between the quartz and the Au can be estimated at approximately $1 \mathrm{~nm}$. This is somewhat thicker than 
might be expected from a monolayer coverage but here the transition between quartz, MPTS and Au layers is also quite broad, indicating a degree of surface roughness in excess of the MPTS layer thickness. These features, described above, of the $S L D(z)$ profile are common to all three traces, Figure $\mathbf{3 b}$, consistent with the expectation that the underlying electrode substrate is the same for all.

In the absence of a copper film, Figure $3 \mathbf{b}$ trace $t=0$, at values of $z>50 \mathrm{~nm}$ the scattering length density drops to a value of $0.5 \times 10^{-6} \AA^{-2}$ corresponding to the bulk solvent medium. The slope of this transition, $\mathrm{d} S L D / \mathrm{d} z$, is a measure of the surface roughness of the $\mathrm{Au} /$ solvent interface with a large value of slope indicating a sharp, smooth interface. In the two subsequent traces, $t=28 \times 10^{3}$ and $t=39.5 \times 10^{3}$, at values of $40<z<80 \mathrm{~nm}$ the value of $S L D$ rises to a plateau at $S L D=6.4 \times 10^{-6} \AA^{-2}$. This is evidence of the formation of a discrete $\mathrm{Cu}$ layer. Copper metal has a bulk SLD value of $6.52 \times 10^{-6} \AA^{-2}$. At increasing values of $z>80 \mathrm{~nm}$, the scattering length density drops again to the bulk solvent value $\left(0.5 \times 10^{-6} \AA^{-2}\right)$. Qualitatively, the slope, $\mathrm{d} S L D / \mathrm{dz}$, of the trace at the end of deposition $\left(t=39.5 \times 10^{3} \mathrm{~s}\right)$ at the $\mathrm{Cu} /$ solvent interface is less steep than that for the $\mathrm{Au} /$ solvent interface. This is evidence that the surface of the growing copper is rougher than that of the Au electrode substrate.

\section{Time-resolved NR data for copper metal deposition}

Films of $\mathrm{Cu}$ were subsequently grown using the three electrochemical control methods, PS $(E=-0.6 \mathrm{~V}, 5 \mathrm{hrs}), \mathrm{GS}\left(i=-330 \mu \mathrm{A}\right.$ equivalent to $-11.8 \mu \mathrm{A} \mathrm{cm}{ }^{-2}$ where the initial applied potential was $-0.5 \mathrm{~V}$ increasing to $-1.0 \mathrm{~V}$ at the end) and PD (linear scan from $E_{1}=0 \mathrm{~V}$ to $E_{2}=-0.8 \mathrm{~V}$ at a potential scan rate, $v=0.02 \mathrm{mV} \mathrm{s}^{-1}$ ) during event mode acquisition of time-resolved neutron reflectivity data. The native $R(Q, t)$ data for the entire growth process are shown as 3D surface plots, Figure $4 \mathbf{a}, \mathbf{b}$ and $\mathbf{c}$. The fitted $\operatorname{SLD}(z, t)$ are presented as discrete slices for clarity in Figure $\mathbf{4 d}$, e and $\mathbf{f}$. Reflectivity fringes can be seen clearly in all the NR data and along with time and potential dependent trends. In the PS and GS data, Figure 4a and Figure 4c, it can be seen that the fringe period decreases with increasing time, indicating the increase in thickness of the Cu layer. The corresponding fitted $\operatorname{SLD}(z, t)$ profiles, Figure $\mathbf{4 d}$ and Figure $\mathbf{4 f}$, show that the initial electrode substrates are very similar (MPTS, Au) in profile and then clearly show sequential growth of the Cu layer over time. In PS data set, Figure $4 \mathbf{d}$, the value 
of SLD corresponding to the Cu layer is approximately $5.8 \times 10^{-6} \AA^{-2}$. This is somewhat lower than the value for the bulk metal $\left(6.58 \times 10^{-6} \AA^{-2}\right)$, suggesting that the coating is less dense than the bulk $\mathrm{Cu}$. This is indicative of porosity and solvent content, since the SLD value for the solvent $\left(0.5 \times 10^{-6} \AA^{-2}\right)$ is much lower than that of the $\mathrm{Cu}$.

The data in Figure $\mathbf{4 b}$, are presented as a function of potential, rather than time, because in this case the electrochemical control was potentiodynamic. This raw data set ${ }^{33}$ is represented for comparison purposes. Here the potential scan rate was $v=0.02 \mathrm{mV} \mathrm{s}^{-1}$. Thus, the potential scan from 0 to $-800 \mathrm{mV}$ encompassed a total time scale of $40 \times 10^{3} \mathrm{~s}$. Here no change in the fringe frequency is visible between $0 \mathrm{~V}$ and $-0.3 \mathrm{~V}$. However, at more negative potentials, close to the onset of the reduction wave in the C.V. (Figure 1), the fringe spacing contracts (layer thickness increases; see above for comment on reciprocal space) as the potential is increased in the cathodic sense. This clearly indicates $\mathrm{Cu}$ film formation and growth and this is also indicated in the fitted $\operatorname{SLD}(z, t)$ data. Note that the time scale for the plot presented in Figure 4 e starts at $t=18 \times 10^{3} \mathrm{~s}$ (equivalent to $-370 \mathrm{mV}$ in the voltammetric scan). This corresponds to the time during the voltammetric scan at which sufficient cathodic potential is reached to drive the reduction process.

Interpretation of the fitted $\operatorname{SLD}(z, t)$ data reveals that the thickest film is seen in the case of PD deposition (Figure 4e), whilst the thinnest film is seen in the GS data (Figure 4f). The shape of the $\operatorname{SLD}(z, t)$ profile at the end of the GS experiment (Figure $4 \mathrm{f}$ ) indicates that the copper/electrolyte interface is rougher than in either of the other two data sets.

The fitted parameters for the film as grown at the end of the experiment, taken from the data in Figure 4, are shown in Table 1. These include the total charge passed during deposition, the final film thickness, final surface roughness, and final solvent content. The film deposited with GS control is the roughest but also the most dense. Both films deposited using potential control, PS and PD, show significant solvent content. The current efficiencies of the three control functions can be estimated by using Faraday's law taking into account the total charge consumed during growth, the final film thickness (determined by NR data fitting), Table 1, and the bulk density of the metal. The growth charge (repeated from Table 1) and the calculated Faradaic current efficiencies are presented in Table 2. The values of current efficiency range quite widely from $62 \%$ for PS control to $85 \%$ for GS control. The value of $85 \%$ is in broad agreement with bulk and microgravimetry determinations reported previously. ${ }^{5}$ 
The highest value of current efficiency also occurs for the coating with the least solvation (i.e. where the bulk density most closely matches the density of the grown metal film). The values of current efficiency observed for the potential control methods are lower than expected for these metals, however, this may be a consequence of the low current density and long time scales of these experiments. The long time scales will exacerbate the contributions of electrolyte degradation and reduction of adventitious water and dissolved oxygen. The background $\mathrm{CV}$, Figure 1c, indicates that metal ion reductions occur well within the solvent limits and that the electrolyte is relatively free contaminants.

\section{Time-resolved NR data for silver metal deposition}

Films of Ag were similarly grown using the three electrochemical control methods, PS $(E=-$ $0.2 \mathrm{~V}$ for $4 \mathrm{hrs}$ ), GS ( $i=-120 \mu \mathrm{A}$ equivalent to $-4.3 \mu \mathrm{A} \mathrm{cm}^{-2}$, with an initial applied potential of $-0.47 \mathrm{~V}$ and a final potential of $-1.0 \mathrm{~V}$ ) and PD (linear scan from $E_{1}=0.2$ to $E_{2}=-0.3 \mathrm{~V}$ at a potential scan rate, $v=0.02 \mathrm{mV} \mathrm{s}^{-1}$ ) during event mode acquisition of time-resolved neutron reflectivity data. The native $R(Q, t)$ data are shown as 3D surface plots, Figure $\mathbf{5 a}, \mathbf{b}$ and $\mathbf{c}$. The fitted $S L D(z)$ are presented as discrete slices for clarity in Figure $\mathbf{5 d} \mathbf{d}$, e and $\mathbf{f}$.

The $R(Q, t)$ profiles for the silver deposition show similar trends to those shown in Figure 4, however, the fringes are less well defined. This is because the neutron reflectivity contrast (i.e. difference in SLD value) between the $A u\left(4.49 \times 10^{-6} \AA^{-2}\right)$ and $A g\left(3.47 \times 10^{-6} \AA^{-2}\right)$ is smaller in magnitude than that between $\mathrm{Au}\left(4.49 \times 10^{-6} \AA^{-2}\right)$ and $\mathrm{Cu}\left(6.52 \times 10^{-6} \AA^{-2}\right)$. This emphasises the need for data fitting procedures. Nonetheless the outcomes of the fitted data in all three cases, Figure $\mathbf{5 d} \mathbf{d}-\mathbf{f}$, clearly show the emergence of the growing Ag film. This is most obviously visible in the fitted PS data, Figure $5 \mathbf{d}$, where the plateau in the $\operatorname{SLD}(z)$ trace at long times $(t>$ $\left.6 \times 10^{3} \mathrm{~s}\right)$ at $c a . S L D=3.5 \times 10^{-6} \AA^{-2}$, indicates the presence of the Ag layer. The value of SLD in this layer (being close to the bulk value for $\mathrm{Ag}$ ) also indicates that the Ag layer produced by this method is dense and uniform in composition. In all three deposition cases a qualitative inspection of the fitted data indicates that the $\mathrm{Ag} /$ electrolyte interface at the end of film growth is rougher than the Au electrode surface. 
The fitted parameters for the Ag film as grown at the end of the experiment, taken from the data in Figure 5, are shown in Table 1. The roughness at the end of film growth was very similar in each case but the most dense and uniform film was obtained under PS control.

The Faradaic current efficiency data were calculated, as for $\mathrm{Cu}$ (above), and are shown in Table 2. Here the values of current efficiency are similar to $\mathrm{Cu}$ and consistent with expectation. There is no discernable trend in these data but here, unlike the Cu case, the best current efficiency is demonstrated un PS control. A common feature of these data for both $\mathrm{Ag}$ and $\mathrm{Cu}$ is that the best current efficiency is observed for the coatings that show the least solvent content.

\section{Trends in time-resolved growth parameters.}

Growth parameters including thickness, roughness and solvation, were extracted from the fitted data for both metals for every recorded reflectivity profile, $R(Q)$, during the deposition experiment. These are presented graphically as a function of time for $\mathrm{Cu}$ and $\mathrm{Ag}$ in Figure 6 and Figure 7 respectively.

Thickness: $\quad$ For $\mathrm{Cu}$ growth, Figure $6 \mathrm{a}$, shows the thickness of the deposit as a function of time, $z(t)$. Here it can be seen that although the final film thickness achieved for each of the 3 methods is quite different (see Table 1), the growth profiles and rates are very similar. At short times the $z(t)$ profile depends on control function as a consequence of differences in nucleation; this is most marked for GS control. At longer times, the three $z(t)$ profiles converge and the only difference between the experiments is how far the experiments traverse this curve. For Ag growth, Figure 7a, the trends are slightly different. Here, at times $t>3000 \mathrm{~s}$, the growth rates of the films in all three experiments was very similar, however, in the early phase of deposition, $t<2000 \mathrm{~s}$, the rate of growth was much faster for the experiment under potentiostatic control. This resulted in a much thicker coating for the PS method. The other coatings achieved here for the PD and GS experiments were of similar magnitude $(z \approx 35 \mathrm{~nm})$ to those for $\mathrm{Cu}$, Figure $6 \mathrm{a}$.

Roughness: Copper growth showed considerable sensitivity of the roughness function, $r(t)$, to deposition method, Figure $\mathbf{6 b}$, with PS control producing the smoothest films. In the initial stages of growth ( $<2000 \mathrm{~s}$ ) no distinct layer is recognisable in the $\operatorname{SLD}(z, t)$ profiles, 
Figure 4 d,e,f. Instead, the change from Au to solvent SLD becomes more gradual before a distinct Cu layer can be recognised at larger thicknesses (longer times). This indicates an initial region with reduced $S L D$ most likely due to discontinuous film growth comprising $\mathrm{Cu}$ nucleation sites and bare Au substrate exposed to solvent. At later times the roughness for each of the three coatings remains quite stable with time. This suggests that the morphology of the growing $\mathrm{Cu} /$ solution interface is dominated by the early formative processes. The relatively high roughness of the coatings grown under GS and PD control may be due to high rates of nucleation at short times, in the case of GS control, and high rates of nucleation later in the film growth in the case of PD control.

In the case of $\mathrm{Ag}$ deposition, Figure $\mathbf{7 b}$, the magnitude of roughness determined for the coating was consistently larger than those for $\mathrm{Cu}$ under similar growth conditions, however, the trends in roughness during growth were very similar for all three deposition methods.

Solvation: The very high levels of solvation obtained in the fits for the initial stages of $\mathrm{Cu}$ growth using the PD and GS methods are due to a strong correlation with the roughness values at this stage (see above), but as growth progresses the film becomes more dense and the solvation levels reach between 0 and 5 vol.\%. This is consistent with a rapid nucleation model where metal nuclei form in large numbers at the surface. Our previous studies in DES media have shown that nucleation mechanism is sensitive to deposition conditions, electrolyte formulation and metal speciation. ${ }^{18,37,38}$ Since the neutron interactions are averaged over the illumination area (foot-print) of the beam, then the observed composition will be a mixture of uncoated surface (exposed to solvent) and metal nuclei. The Cu PS solvation data, Figure $\mathbf{6 c}$, show gradually increasing solvation produced by this method. This may be the result of a progressive nucleation and growth regime produced by the gradually increasing applied potential. The equivalent data for Ag growth, Figure 7c, show quite large and erratic variation in solvation for the PD and GS growth. This will be correlated with a rather noisy and increasing roughness, Figure $\mathbf{7 b}$,

but the SLD profiles reveal a somewhat decreased bulk density and larger roughness for films grown by these methods. Potentiostatic growth, on the other hand, produced a film with very low solvent content despite the gradual increase in apparent roughness. The low solvent content exhibited at longer times can be attributed to dominance of the interior composition of the bulk film, whereas the roughness, by definition, represents the exterior of the film. 
In both cases here ( $\mathrm{Cu}$ and $\mathrm{Ag})$, the values of solvation in the range 5-10 vol. \% are quite large. Given the high chloride content of the DES medium this may be an important factor in understanding the potential of these coatings in corrosion protection.

\section{Data fitting and validation}

In considering the outcomes and interpretation of the data fitting described above we have been mindful of the complexity of data fitting algorithms in multi-parameter models. Consequently, we have sought to validate and optimise the outcomes of the fitting in two ways i) by verification using independent measurement where possible and ii) by repetitive and duplicate treatment (fitting) of the data using different modelling tools and a range of starting conditions. Further, the volume of data generated by event mode capture results in very large numbers of data sets. Here we have used both parallel and serial fitting methodologies. Although the parallel and serial fitting software utilise the same numerical algorithms we have sought to establish that the outcomes of the fitting process are not unduly sensitive to either the initial conditions or methods.

Metrology: Thickness and roughness parameters were measured independently at the end of selected experiments using ex-situ atomic force microscopy (AFM). ${ }^{\ddagger}$ For these measurements, the cell was dismantled and the electrode substrate washed with water and dried with ethanol and acetone. The thickness and roughness were determined at three locations spanning the edge and middle of the film and the average was then reported. The AFM images of representative $\mathrm{Cu}$ and $\mathrm{Ag}$ surfaces deposited under PS control are shown in Figure 8. The values for thickness and roughness are also presented in Table 1, for comparison, alongside the values determined from data fitting of the $R(Q)$ NR profiles. In the case of Ag deposition under PS control, the ex-situ AFM measurements are in very good agreement with the values obtained by fitting of the NR data. In the case of $\mathrm{Cu}$, the values obtained for both thickness and roughness from ex situ AFM are significantly larger than those determined from fitting of the NR data. In the case of the roughness, it can be seen from Figure 8a that the surface of the $\mathrm{Cu}$ is quite inhomogeneous on the scale of the AFM image

\footnotetext{
‡ Any surface oxide layer formed in this period of time would be very thin in comparison to the total film thickness.
} 
$(3 \times 3 \mu \mathrm{m})$ showing large nuclei separated by smoother regions. The roughness determined from AFM is measured over the area of this image $\left(9 \mu \mathrm{m}^{2}\right)$ whereas the roughness determined from the NR data is averaged over a much larger are defined by the foot print of the beam ( 2 $\mathrm{cm}^{2}$ i.e. $200 \times 10^{6} \mu \mathrm{m}^{2}$ ). The film thickness determined from AFM was measured across a line trace traversing a purpose made scratch in the film. In this case the large value of the AFM thickness compared to the NR data may be the result of the line trace incorporating the $\mathrm{Au}$ substrate (i.e. the scratch may have penetrated to the quartz). Additionally, the action of scratching the coating can also cause delamination and peeling of the film that is very difficult to see in the image. Peeling at the measurement edge of the film would result in unpredictable over-estimation of film thickness.

Data processing: To garner confidence in the outcomes of the necessary data fitting processes we conducted parallel fitting procedures of selected sample data sets using two software packages developed by the neutron user's community. These are named RasCal (which functions as a script in MatLab) and MotoFit (which functions within Igor). Both fitting methods use the same numerical processing algorithms and iterative minimisation procedures but the raw data handling and model descriptions are quite different. In particular, the RasCal method treats multiple defined data sets in a parallel fitting procedure whereas MotoFit only operates on a single, $R(Q)$, data set. Consequently, RasCal offers an advantage in the fitting of large time-resolved data sets but the outcomes of both methods for a particular time slice, $R(Q, t)$, should be the same.

The data presented in Figure 9 show the outcomes of one such trial. Here the $R(Q, t)$ data for PS growth of a Ag film were treated independently by three of the authors here (E.L.S, E.J.R.P and R.S.) using the two software packages, RasCal and MotoFit. The initial conditions for the model interfaces were defined independently and separately. The outcomes of this process, Figure 9, show that in this case, the thickness/time, $z(t)$, data derived from the NR profiles were qualitatively indistinguishable and quantitatively within experimental error.

\section{Conclusions}

We set out to study the electrochemical deposition of copper and silver as thin-film metals onto a gold electrode substrate from a deep eutectic solvent using potentiodynamic (PD), 
potentiostatic (PS) and galvanostatic (GS) electrochemical control functions. In particular, we have utilised new and novel developments in neutron reflectivity methods to acquire realtime data for the growing metal films. Event mode capture of neutron scattering events, as a function of momentum transfer vector, $Q$, during electrochemical growth has enabled timeresolved measurement of the neutron reflectivity, $R(Q)$, profiles of the growing metal films. Subsequent fitting and iterative optimisation of these, $R(Q, t)$, data using a multilayer model has yielded the thickness, roughness and relative density (spatially resolved solvent content) of the metal film during growth.

The first significant outcome of these data is that the different electrochemical growth methodologies, PS, PD and GS, exhibit subtly different trends in thickness, roughness and solvation. Both metals exhibit non-linear growth as a function of time such that growth rate slows at longer times. The electrochemical current data (in the form of linear $i\left(t^{-1 / 2}\right)$ plots) are indicative of mass transport limited behaviour. Film growth (represented by linear $z\left(t^{1 / 2}\right)$ plots) is fully consistent with this. Some deviation from this is observed at early times, as a consequence of nucleation kinetics, the nature of which is control function dependent.

Second, turning to the deposition charge efficiency, the NR data show that for both metals the charge efficiency of deposition is highest where the coating has least solvent content. In the Cu case this is delivered by GS control whereas in the Ag case this is under PS control. This is where the density of the coating most closely approximates to the density of the bulk metal. The overall thickness of films obtained during the experimental time scales were similar for both metals. In the case of Cu deposition, potential control methods produced very similar growth rates but growth under GS control was initially very slow. This was not the same for Ag deposition, where PS control produced very fast initial growth.

Third, solvent incorporation in both metal films in this DES medium (Ethaline 200) varies measurably with control function. This is a significant insight in situations where the metals could be used in an environment where corrosion protection is required, or where pure, dense and uniform coatings are specified. The extent of solvent incorporation is strongly correlated with surface roughness in the early phases of growth. Potentiostatic deposition of $\mathrm{Ag}$ and potentiodynamic deposition of $\mathrm{Cu}$ gave metal deposits with low solvent content.

Fourth, film roughness effects differ for silver and copper. Silver films show an increasing roughness trend with time but these trends are largely independent of growth method. In 
contrast, the roughness of copper films, grown under similar conditions, show a strong dependency on growth method with PS methods producing smoothest films. The overall roughness was generally greater for the silver films. Hence it is an important conclusion here that the Cu films are generally lower in surface roughness but that Cu growth is more sensitive to the control conditions. These conclusions are confirmed by ex-situ measurement using AFM.

Finally, from a methodological perspective, we have established a robust and stable approach to fitting and interpretation of the large number of data sets generated by event mode capture. The $N R R(Q, t)$ data were fitted using both parallel (RasCal) and serial (MotoFit) methodologies utilising a variation of initial starting conditions and models. Gratifyingly, the different methodologies and starting conditions yield a very consistent picture of metal film growth in that the outcomes from all data fitting are qualitatively indistinguishable and quantitatively within experimental error.

In the modelling and fitting of the NR data we have considered the consequences of intermetallic diffusion and mixing at the interfaces, however, separate galvanostaic experiments on the stripping of both $\mathrm{Ag}$ and Cu layers from gold substrates using the so-called STEP method (Simultaneous Thickness Electrochemical Potential) shows no evidence to indicate formation of solid solution zones or intermetallic phases on these time scales.

In further work and ongoing studies, we are using these methods to characterise the structure and stability of multi-layer metal interfaces (including $\mathrm{Cu} / \mathrm{Ag}, \mathrm{Au} / \mathrm{Pd}$ and $\mathrm{Cu} / \mathrm{Ni} / \mathrm{Au}$ ) that are key to the function of PCB assemblies. The methods we describe here are especially well suited to measuring of inter-diffusion and solid-state mixing between adjacent electrochemically deposited layers. The rate and extent of such processes determine the physical characteristics, mechanical strength and durability of subsequent assemblies (electronic components) fabricated on these substrates.

\section{Acknowledgements}

The authors gratefully acknowledge the STFC for provision of beam time at ISIS (Rutherford Appleton Lab. Oxford; experiment No's: RB1320411 and RB1510528) on OFFSPEC. The raw data for these experiments are made available at DOI: 10.5286/ISIS.E.47624376 and DOI: 
10.5286/ISIS.E.59115258 for experiment RB1320411 and at DOI: 10.5286/ISIS.E.58449398 for experiment RB1510528. KSR also thanks Innovate UK (Manufacturing Electronic Systems of the Future) for funding under the MACFEST project (Project No: 102020). 


\begin{tabular}{|c|c|c|c|c|c|c|c|}
\hline \multirow{3}{*}{ Metal } & \multicolumn{2}{|c|}{ Electrochemistry } & \multicolumn{3}{c|}{ NR } & \multicolumn{2}{c|}{ AFM } \\
& $\begin{array}{c}\text { Deposition } \\
\text { method }\end{array}$ & $\begin{array}{c}\text { Growth } \\
\text { charge } \\
/ \mathrm{mC} \mathrm{cm}^{-2}\end{array}$ & $\begin{array}{c}\text { Thickness } \\
/ \mathrm{nm}\end{array}$ & $\begin{array}{c}\text { Roughness } \\
/ \mathrm{nm}\end{array}$ & $\begin{array}{c}\text { Solvation } \\
\%\end{array}$ & $\begin{array}{c}\text { Thickness } \\
/ \mathrm{nm}\end{array}$ & $\begin{array}{c}\text { Roughness, } \\
R_{\mathrm{a}} / \mathrm{nm}\end{array}$ \\
\hline \multirow{3}{*}{$\mathrm{Cu}$} & $\mathrm{PS}$ & 113 & $25.6 \pm 0.3$ & $4.7 \pm 0.2$ & $8.3 \pm 1$ & $77 \pm 4$ & 15.9 \\
\cline { 2 - 8 } & $\mathrm{PD}$ & 136 & $36.9 \pm 0.5$ & $6.6 \pm 0.2$ & $5.3 \pm 1$ & -- & - \\
\cline { 2 - 8 } & $\mathrm{GS}$ & 63 & $19.4 \pm 2.2$ & $7.1 \pm 0.7$ & $0.0 \pm 2$ & -- & \\
\hline \multirow{3}{*}{$\mathrm{Ag}$} & $\mathrm{PS}$ & 68 & $59.1 \pm 0.3$ & $10.7 \pm 0.5$ & $0.0 \pm 1$ & $66 \pm 2$ & 7.7 \\
\cline { 2 - 8 } & $\mathrm{PD}$ & 56 & $36.1 \pm 0.1$ & $13.0 \pm 0.1$ & $3.3 \pm 1$ & - & - \\
\cline { 2 - 8 } & $\mathrm{GS}$ & 55 & $35.4 \pm 0.1$ & $9.9 \pm 0.3$ & $6.9 \pm 1$ & - & - \\
\hline
\end{tabular}

Table 1 Comparison of film properties for $\mathrm{Cu}$ and $\mathrm{Ag}$ films grown using potentiostatic (PS), potentiodynamic (PD) and galvanostatic (GS) methods. The films were quantified in terms of total charge consumed during deposition as well as the thickness and roughness determined using neutron reflectivity (NR) and atomic force microscope (AFM) methods. Solvent content was also determined using NR methods. These are the fitted values from the final time-slice of the data presented in Figure 4 and Figure 5.

\begin{tabular}{|c|c|c|c|}
\hline \multirow[b]{2}{*}{ Metal } & \multicolumn{3}{|c|}{ Electrochemistry } \\
\hline & $\begin{array}{l}\text { Deposition } \\
\text { method }\end{array}$ & $\begin{array}{l}\text { Growth charge } \\
\qquad / \mathrm{mC} \mathrm{cm}^{-2}\end{array}$ & $\begin{array}{l}\text { Faradaic current } \\
\text { efficiency }\end{array}$ \\
\hline \multirow{3}{*}{$\mathrm{Cu}$} & PS & 113 & $62 \%$ \\
\hline & PD & 136 & $74 \%$ \\
\hline & GS & 63 & $84 \%$ \\
\hline \multirow{3}{*}{$\mathrm{Ag}$} & PS & 68 & $81 \%$ \\
\hline & PD & 56 & $60 \%$ \\
\hline & GS & 55 & $60 \%$ \\
\hline
\end{tabular}

Table 2 Faradaic current efficiency data calculated from the consumed growth charge and the film thickness determined by fitting of the NR data (Table 1). 


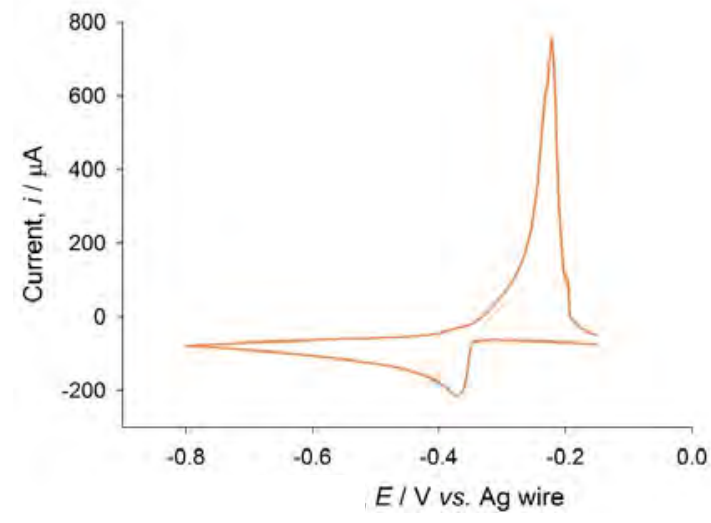

(a)

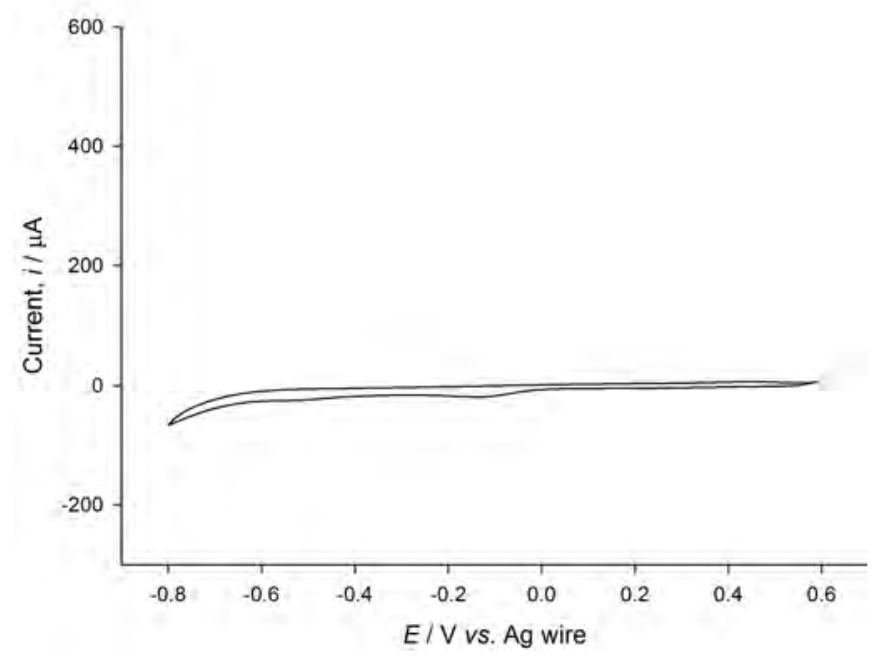

(c)

(b)

Figure 1

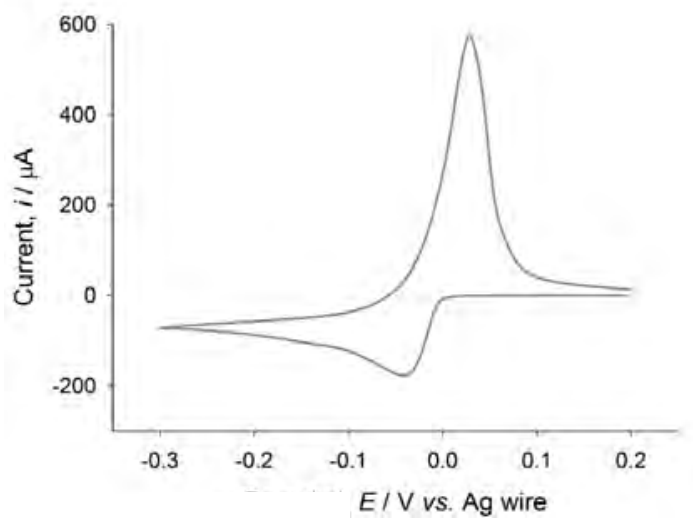

Cyclic voltammograms in the Deep Eutectic Solvent, Ethaline (choline chloride 1:2 ethylene glycol), at $0.02 \mathrm{mV} \mathrm{s}^{-1}$; (a) $10 \mathrm{mM} \mathrm{CuCl}_{2}$ between 0 and $-0.8 \mathrm{~V}$ and $(b) 10$ $\mathrm{mM} \mathrm{AgCl}$ between 0.2 and $-0.3 \mathrm{~V}$; These data were recorded in the neutron cell, $\mathrm{Au}$ electrode area $28 \mathrm{~cm}^{2}$. The $\mathrm{CV}$ of the background electrolyte, $(c)$, is included for clarity. 


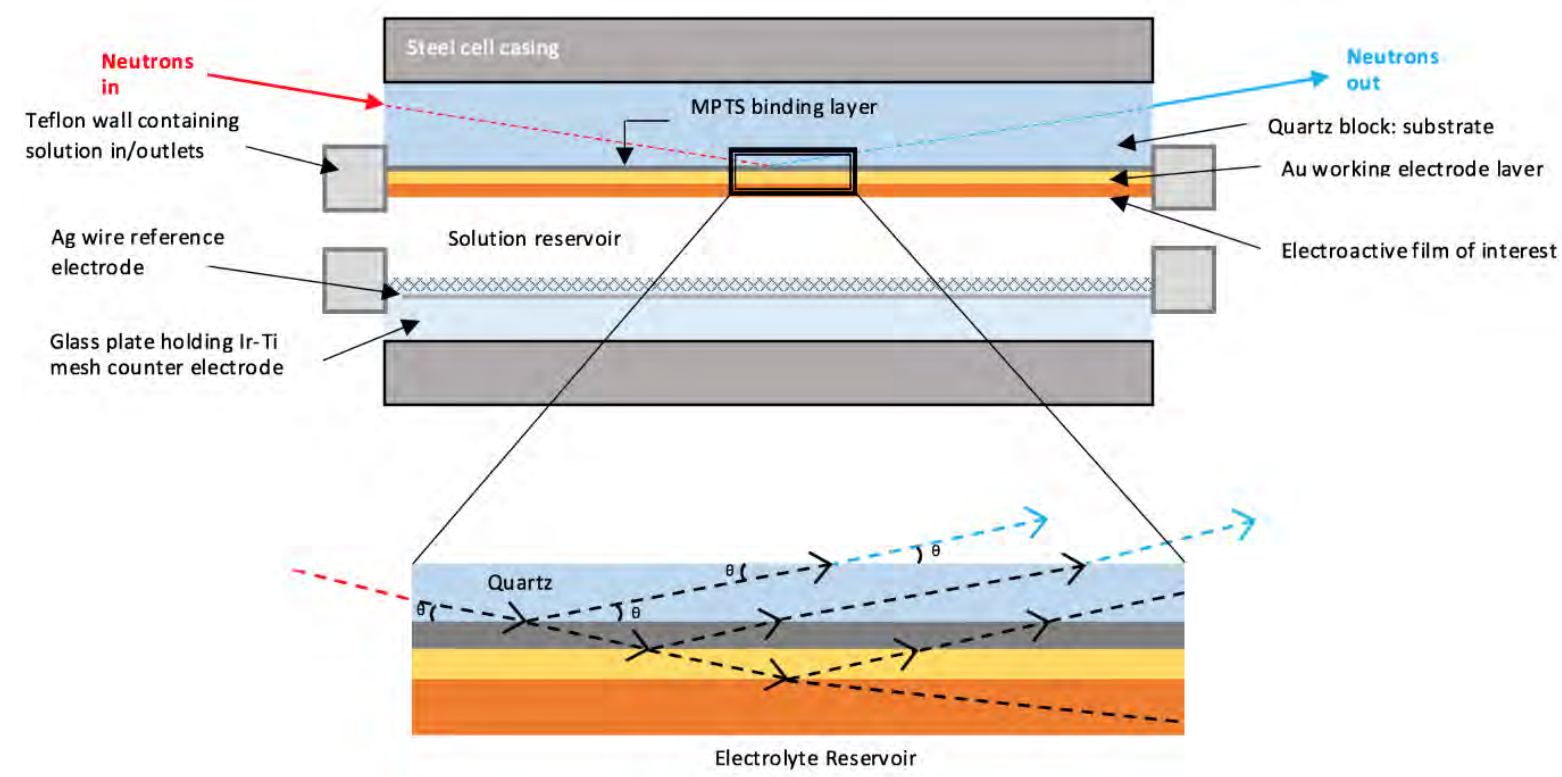

Figure 2 Experimental diagram of the neutron cell, electrodes \& neutron path. The insert shows an expanded view of the solid/solid and solid/liquid interfaces and associated neutron beam paths that generate the observed neutron reflectivity interference fringes. The cell volume was $25 \mathrm{~cm}^{3}$. 


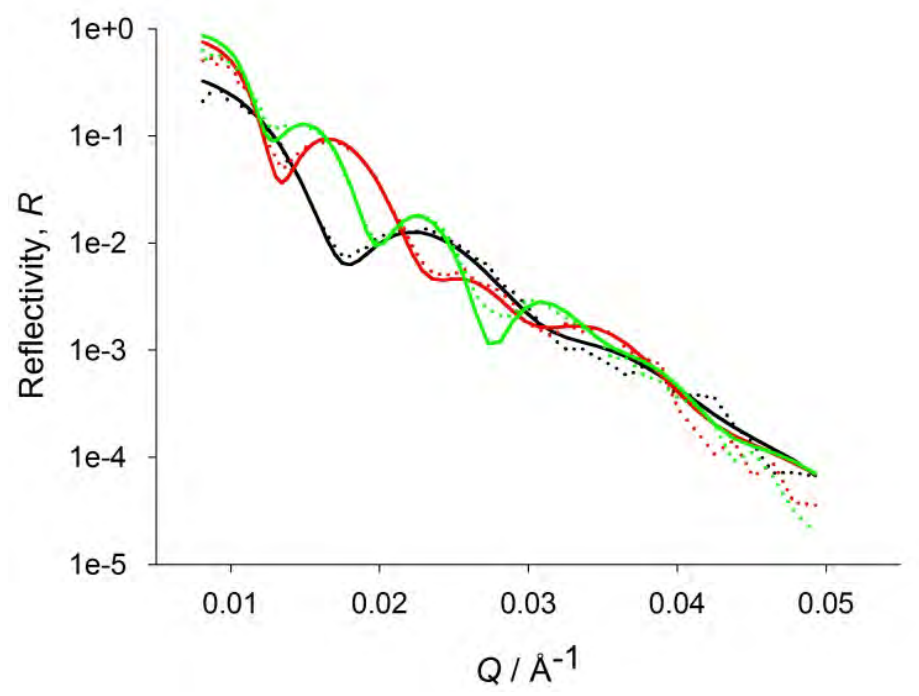

(a)

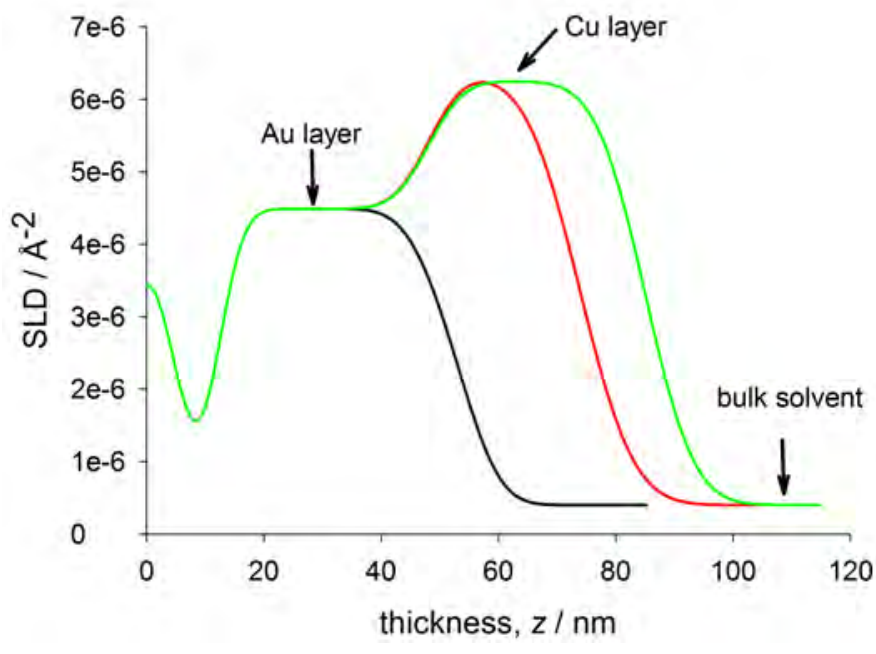

(b)

Figure 3 Sample NR data, $(a)$ Reflectivity, $R(Q)$, plots for the growth (under PD control, by scanning from $0 \mathrm{~V}$ to $-0.8 \mathrm{~V}, v=0.02 \mathrm{mV} \mathrm{s}^{-1}$ ) of a copper thin film; $(b)$ corresponding scattering length density profile, $\operatorname{SLD}(z)$, for the data (part (a)) fitted to a thin film model. The black trace shows the substrate before deposition, $t=0 \mathrm{~s}$, the red trace corresponds to deposition at $t=28.5 \times 10^{3} \mathrm{~s}$ and the green trace is close to the end of the experiment at $t=39.5 \times 10^{3} \mathrm{~s}$. 


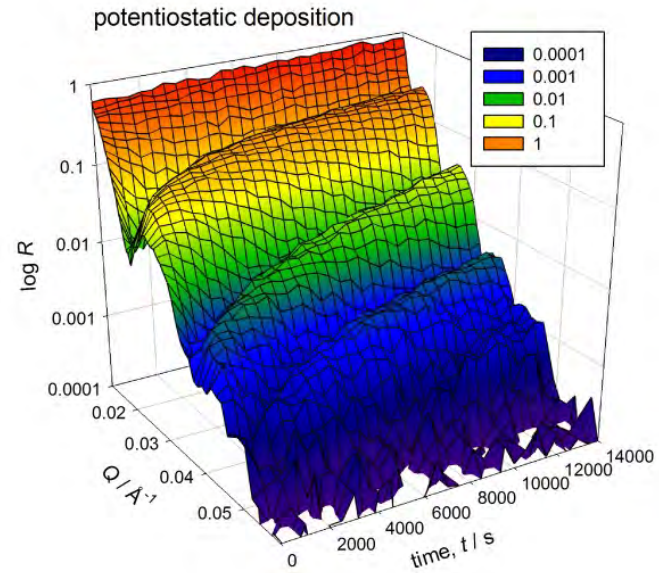

(a)

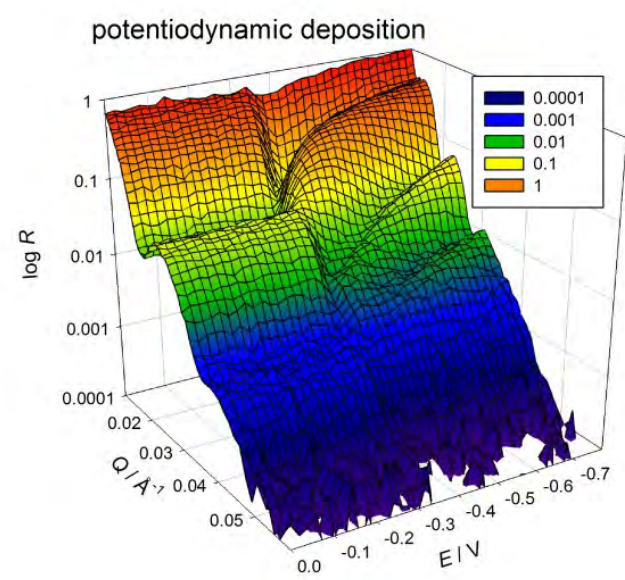

(b)

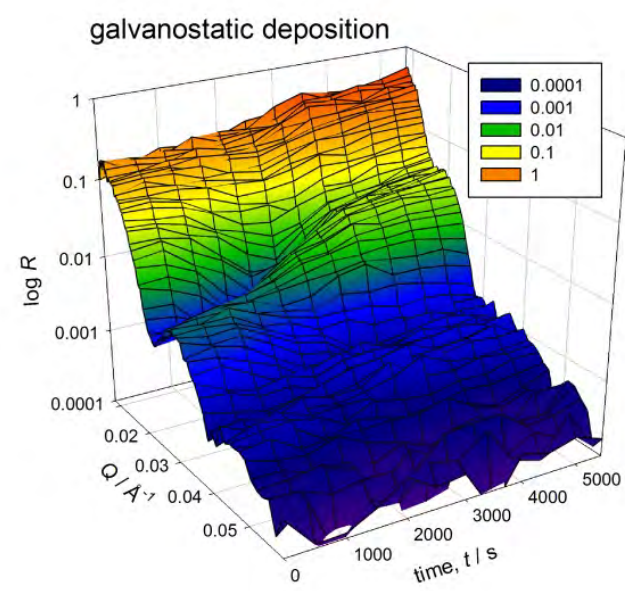

(c)

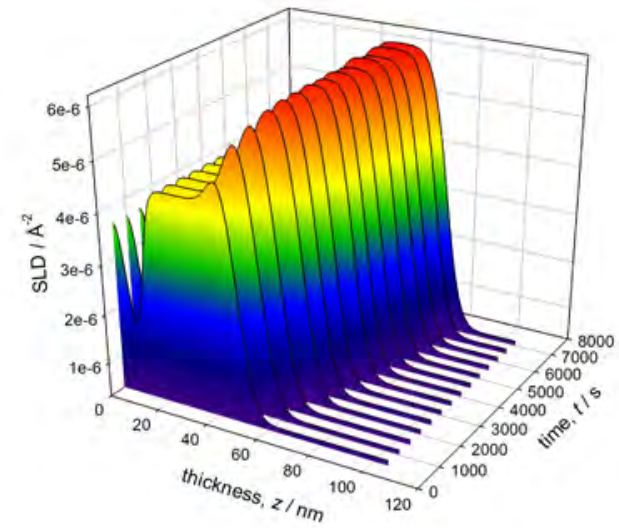

(d)

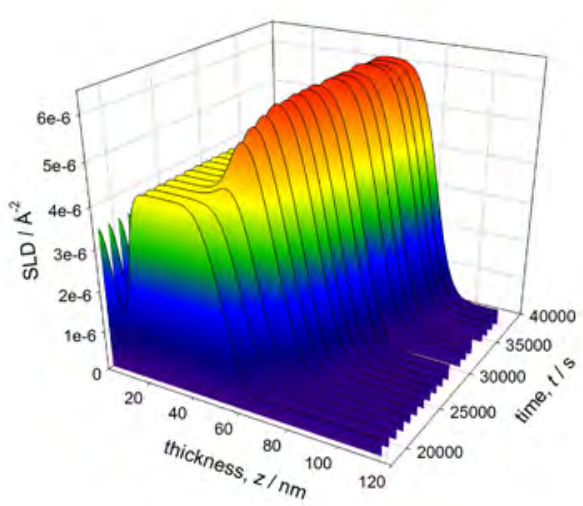

(e)

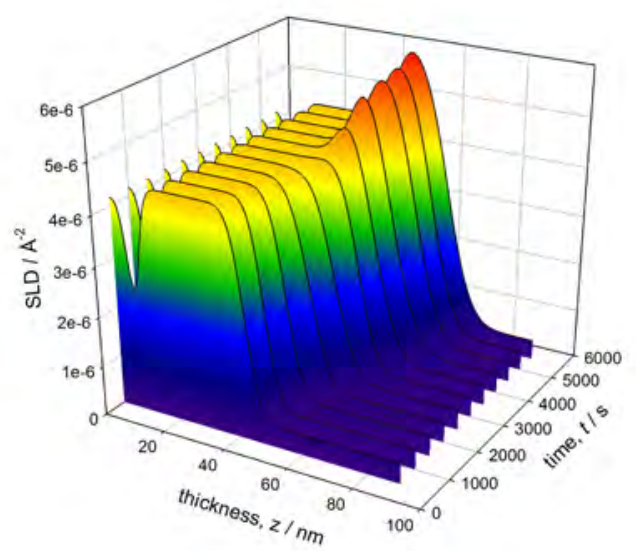

(f)

Figure 4 Dynamic NR profiles of $\mathrm{Cu}$ growth as functions of $\mathrm{Q}$ and time for potentiostatic $(a)$, potentiodynamic $(b)$ and galvanostatic $(c)$ deposition; and the NR models for the dynamic $\mathrm{Cu}$ growth as functions of thickness and time for potentiostatic $(d)$, potentiodynamic $(e)$ and galvanostatic $(f)$ deposition. Inset legends show colour scheme for reflectivity on a log scale. 


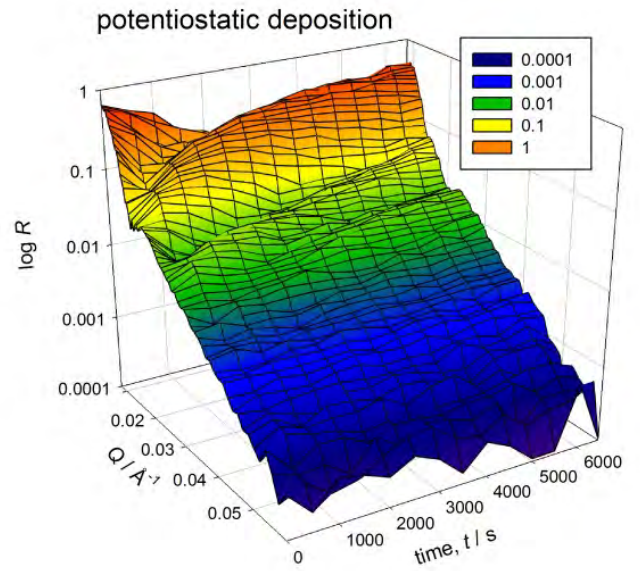

(a)

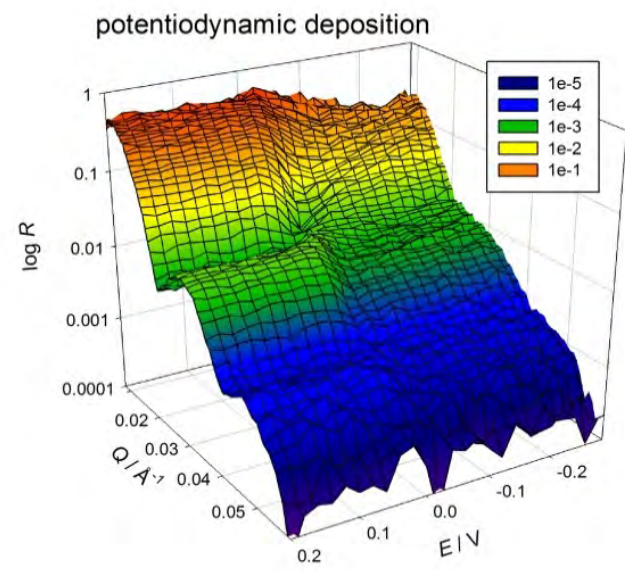

(b)

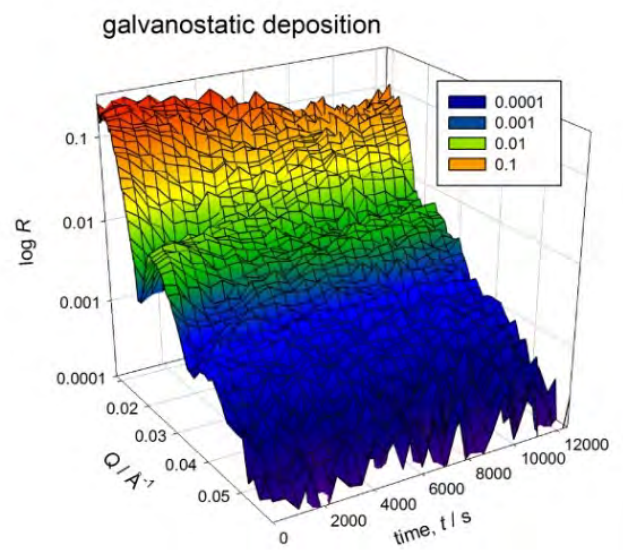

(c)

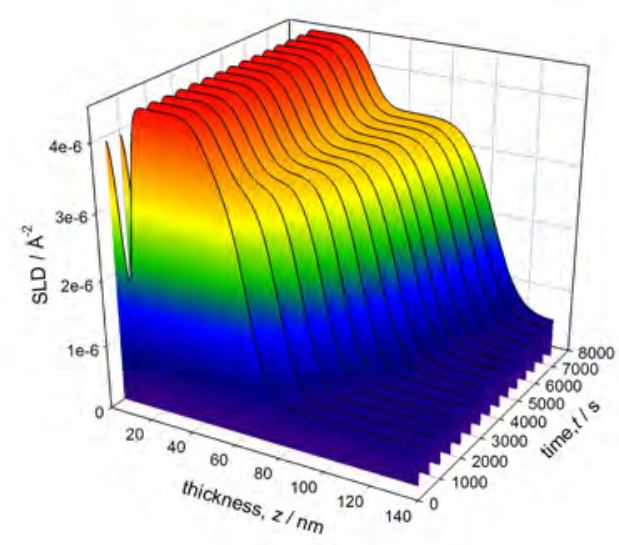

(d)

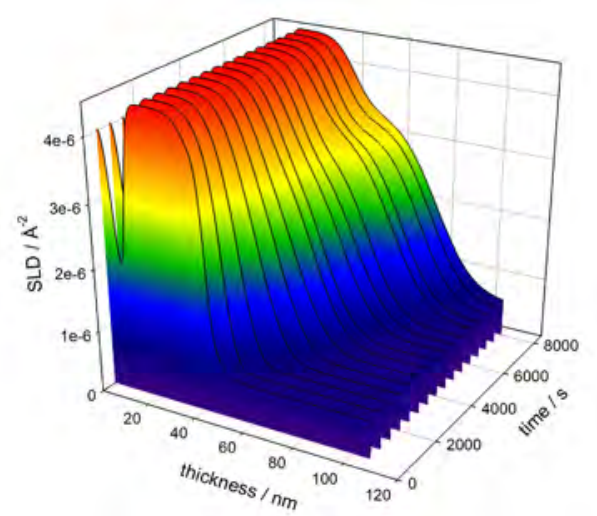

(e)

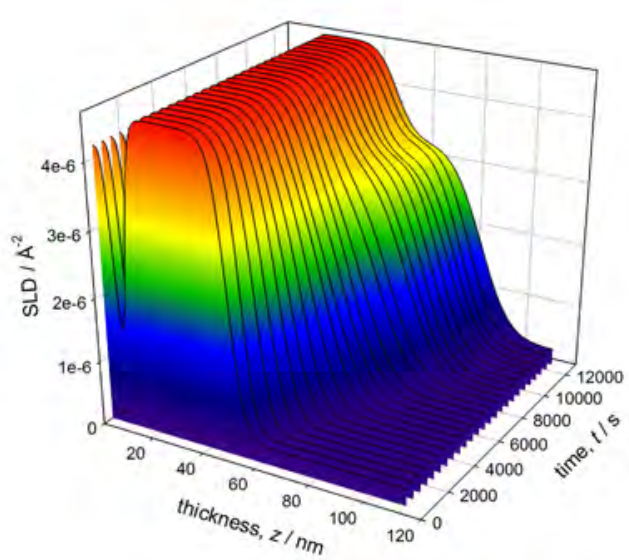

(f)

Figure 5 Dynamic NR profiles of Ag growth as functions of $\mathrm{Q}$ and time for potentiostatic $(a)$, potentiodynamic $(b)$ and galvanostatic $(c)$ deposition; and the NR models for the dynamic Ag growth as functions of thickness and time for potentiostatic $(d)$, potentiodynamic $(e)$ and galvanostatic $(f)$ deposition. Inset legends show colour scheme for reflectivity on a log scale. 


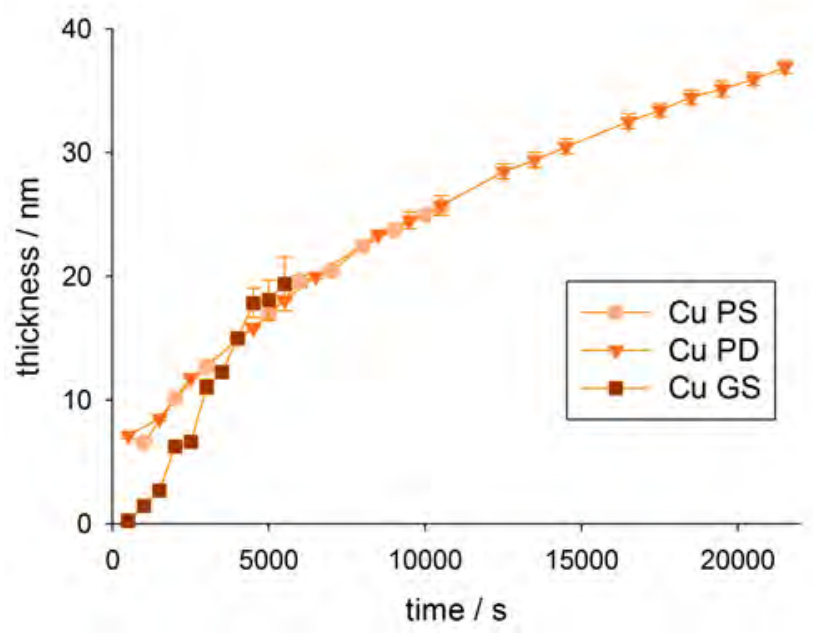

(a)

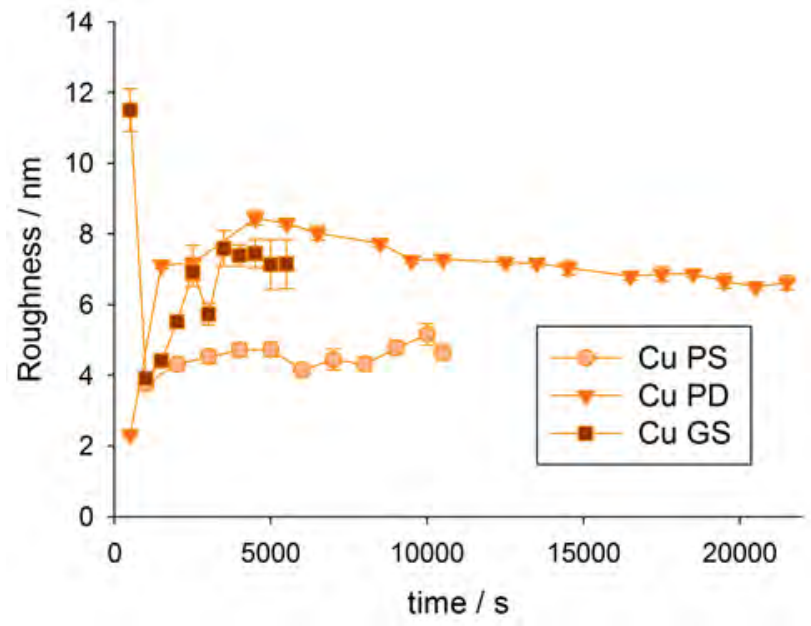

(b)

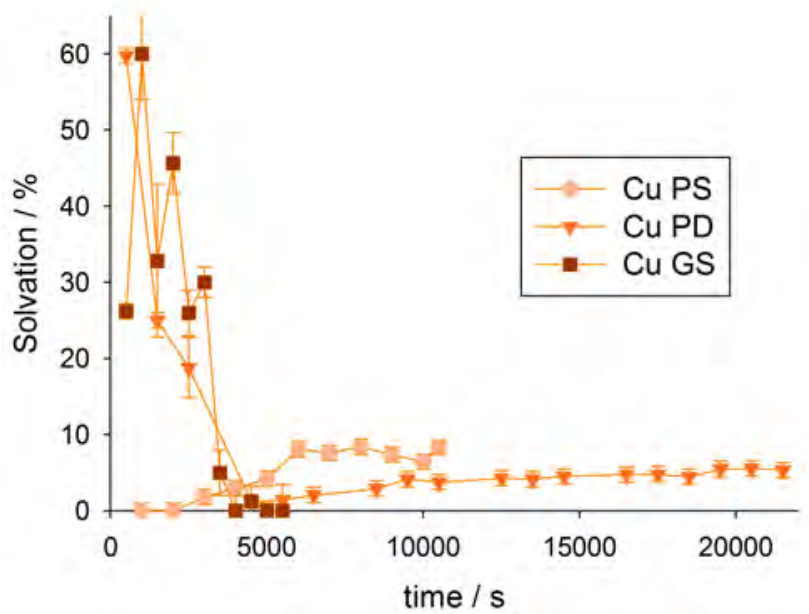

(c)

Figure 6 Time resolved thickness $(a)$, roughness $(b)$ and solvation $(c)$ plots for $\mathrm{Cu}$. Obtained from the fitted (RasCal) data presented in Figure 4. 


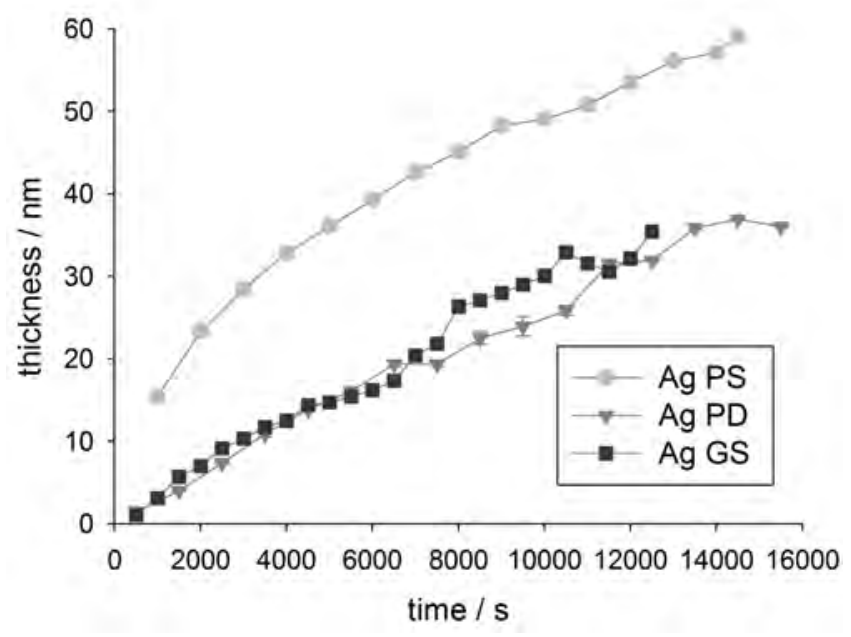

(a)

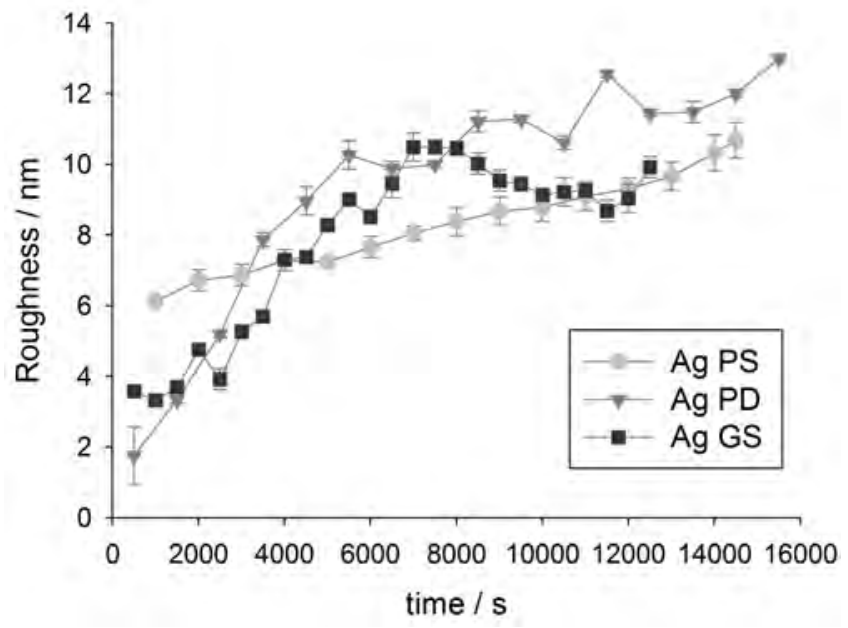

(b)

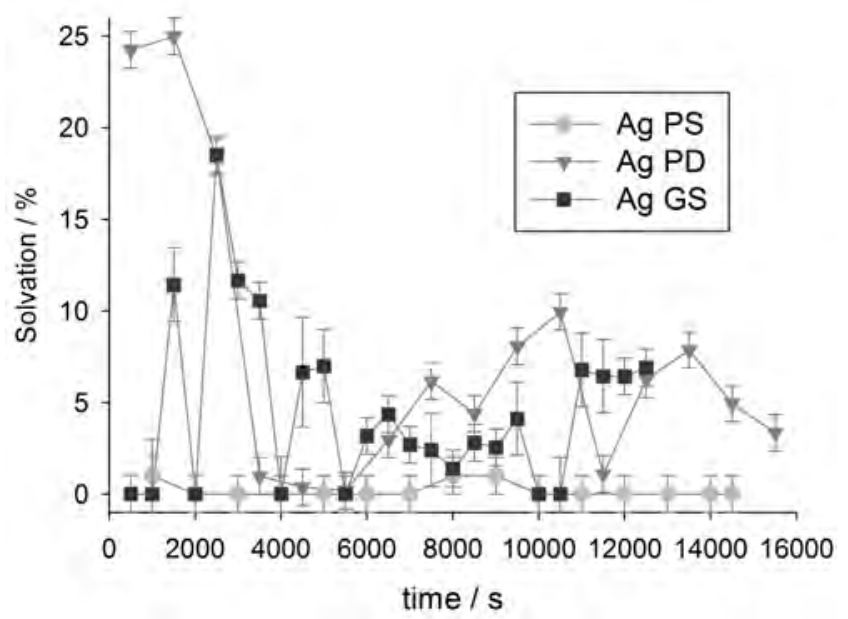

(c)

Figure 7

Time resolved thickness $(a)$, roughness $(b)$ and solvation $(c)$ plots for $\mathrm{Ag}$. Obtained from the fitted (RasCal) data presented in Figure 5. 


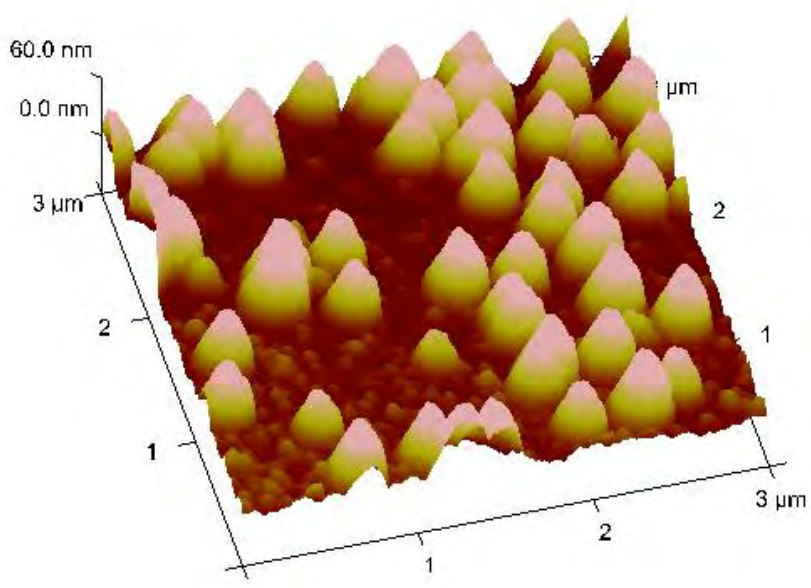

(a)

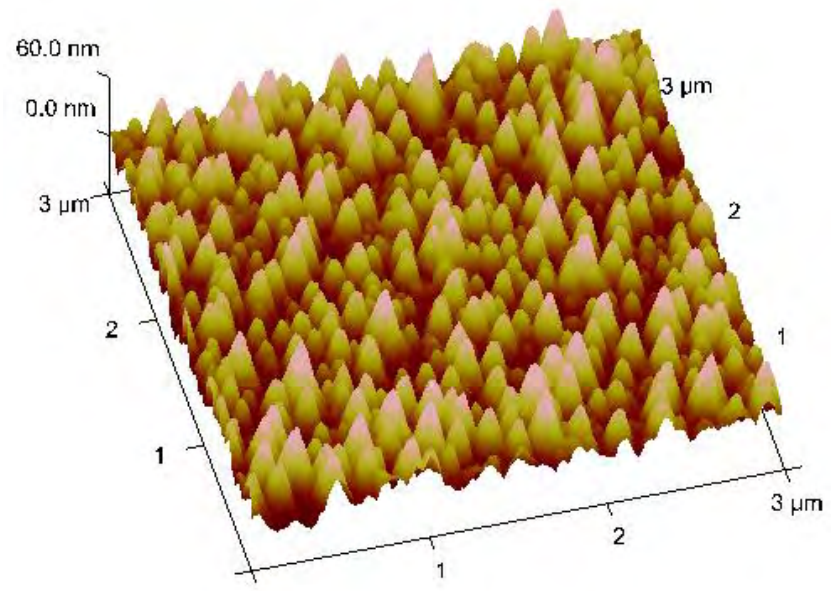

(c)

Figure 8 Representative AFM images for Cu \& Ag grown under PS control; (a) 3D projection for $\mathrm{Cu}$ film, and $b$ ) 3D projection for Ag film. 


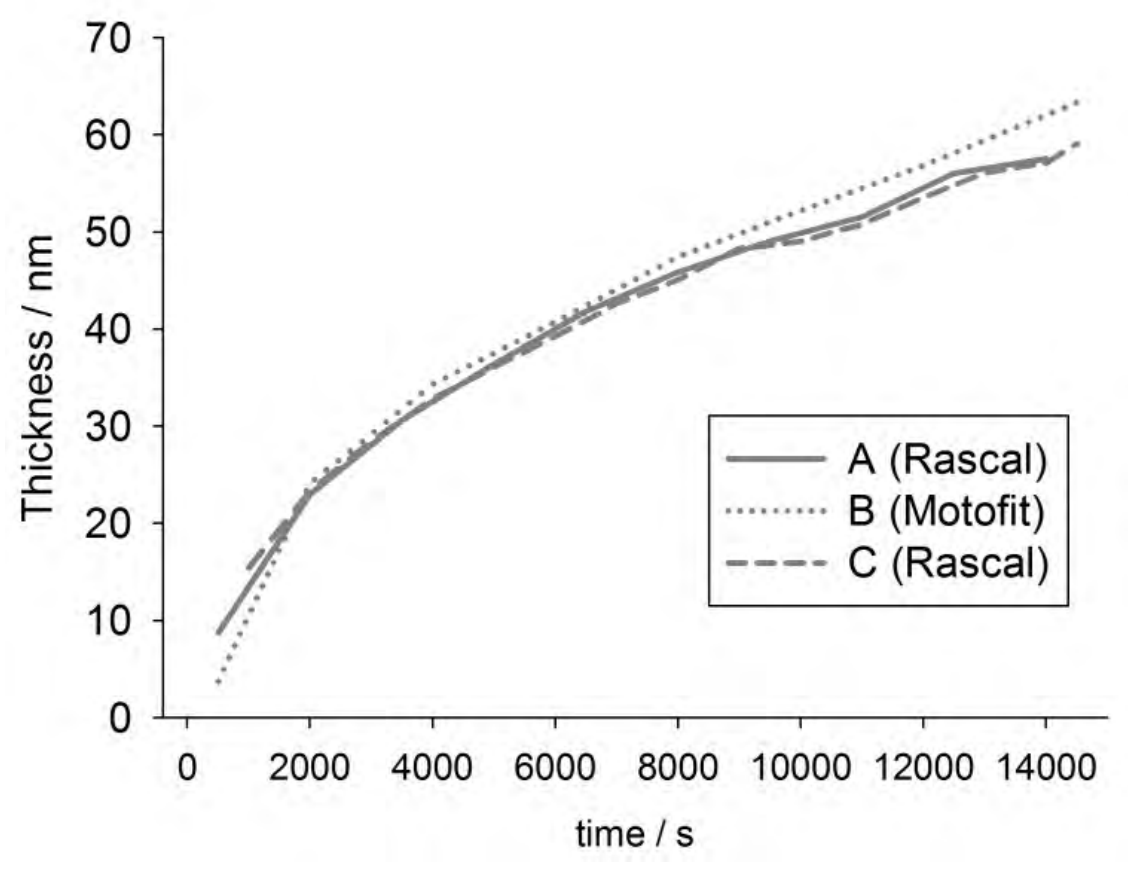

Figure 9 Thickness data vs time, $z(t)$, for PS Ag growth (Figure 5) fitted by three authors here using MotoFit and RasCal software packages (E.J.R.P. RasCal, E.L.S. RasCal, R.S. MotoFit). 


\section{References}

1 H. Yang, X. Guo, N. Birbilis, G. Wu and W. Ding, Applied Surface Science, 2011, 257, 9094-9102

2 J. P. Hallett and T. Welton, Chemical Reviews, 2011, 111, 3508-3576.

3 E. L. Smith, A. P. Abbott and K. S. Ryder, Chemical Reviews, 2014, 114, 11060-11082.

$4 \quad$ P. De Vreese, N. R. Brooks, K. Van Hecke, L. Van Meervelt, E. Matthijs, K. Binnemans and R. Van Deun, Inorganic Chemistry, 2012, 51, 4972-4981.

5 A. P. Abbott, K. El Ttaib, G. Frisch, K. J. McKenzie and K. S. Ryder, Physical Chemistry Chemical Physics, 2009, 11, 4269-4277.

6 A. R. Hillman, K. S. Ryder, C. J. Zaleski, V. Ferreira, C. A. Beasley and E. Vieil, Electrochimica Acta, 2014, 135, 42-51.

7 S. Ghosh and S. Roy, Materials Science and Engineering B-Advanced Functional Solid-State Materials, 2014, 190, 104-110.

8 S. Salome, N. M. Pereira, E. S. Ferreira, C. M. Pereira and A. F. Silva, Journal of Electroanalytical Chemistry, 2013, 703, 80-87.

9 A. P. Abbott, J. C. Barron and K. S. Ryder, Transactions of the Institute Metal Finishing, 2009, 87, 201-207.

10 M. Starykevich, A. N. Salak, D. K. Ivanou, A. D. Lisenkov, M. L. Zheludkevich and M. G. S. Ferreira, Electrochimica Acta, 2015, 170, 284-291.

11 J. Zhang, C. Gu, Y. Tong, J. Gou, X. Wang and J. Tu, RSC Advances, 2015, 5, 71268-71277.

12 E. S. C. Ferreira, C. M. Pereira and A. F. Silva, Journal of Electroanalytical Chemistry, 2013, 707, 52-58.

13 S. Fashu, C.-d. Gu, J.-I. Zhang, M.-I. Huang, X.-I. Wang and J.-p. Tu, Transactions of Nonferrous Metals Society of China, 2015, 25, 2054-2064.

14 A. P. Abbott, G. Capper, K. J. McKenzie and K. S. Ryder, Journal of Electroanalytical Chemistry, 2007, 599, 288-294.

15 F. Liu, Y. Deng, X. Han, W. Hu and C. Zhong, Journal of Alloys and Compounds, 2016, 654, 163170.

16 A. Ballantyne, G. Forrest, M. Goosey, A. Griguceviciene, J. Juodkazyte, R. Kellner, A. Kosenko, R. Ramanauskas, K. S. Ryder, A. Selskis, R. Tarozaite and E. Veninga, Circuit World, 2012, 38(1), 21

17 S. Sigalov, N. Shpigel, M. D. Levi, M. Feldberg, L. Daikhin and D. Aurbach, Anal. Chem., 2016, 88(20), 10151

18 A. P. Abbott, M. Azam, K. S. Ryder and S. Saleem, Anal. Chem., 2013, 85, 6653.

19 A. J. Goddard, R. C. Harris, S. Saleem, M. Azam, C. Hood, D. Clark, J. Satchwell and K. S. Ryder, Trans. IMF, 2017, 95(3) 137.

20 G. Pulletikurthi, M. S. Ghazvini, T. Cui, N. Borisenko, T. Carstens, A. Borodin and F. Endres, Dalton Trans., 2017, 46, 455.

21 A. P. Abbott, J. C. Barron, K. S. Ryder and E. L. Smith, Anal. Chem., 2009, 81, 8466.

22 J. Penfold, R. M. Richardson, A. Zarbakhsh, J.R.P. Webster, D.G. Bucknall, A. R. Rennie, R.A.L. Jones, T. Cosgrove, R. K. Thomas, J. S. Higgins, P. D. I. Fletcher, E. Dickinson, S. J. Roser, I. A. McLure, A. R. Hillman, R. W. Richards, E. J. Staples, A. N. Burgess, E. A. Simister and J.W. White, Faraday Trans., 1997, 93, 3899-3917. 
23 A. R. Hillman, A. Glidle, R. M. Richardson, S. J. Roser, P. M. Saville, M. J. Swann and J. R. P. Webster, J. Am. Chem. Soc., 1998, 120, 12882- 12890.

24 A. R. Hillman, L. Bailey, A. Glidle, J. M. Cooper, N. Gadegaard and J. R. P. Webster, J. Electroanal. Chem., 2002, 532, 269-276.

25 P. M. Saville, M. Gonsalves, A. R. Hillman and R. Cubitt, J. Phys. Chem. B, 1997, 101, 1-4

26 A. Glidle, J. Cooper, A. R. Hillman, L. Bailey, A. Jackson and J. R. P. Webster, Langmuir, 2003, 19, 7746-7753.

27 A. Glidle, L. Bailey, C. S. Hadyoon, A. R. Hillman, A. Jackson, K. S. Ryder, P. M. Saville, M. J. Swann, J. R. P. Webster, R. W. Wilson and J. Cooper, Anal. Chem. 2001, 73, 5596-5606.

28 A. Glidle, C. S. Hadyoon, N. Gadegaard, A. R. Hillman, K. S. Ryder, J. R.P. Webster, J. M. Cooper, R. W. Wilson and R. Cubitt, J. Phys. Chem. B, 2005, 109, 14335.

29 J. F. K. Coopera, K. N. Vyas, N.-J. Steinke, D. M. Love, C. J. Kinane and C. H. W. Barnes, Electrochimica Acta, 2014, 138, 56.

30 I. Burgess, M. Li, S. L. Horswell, G. Szymanski, J. Lipkowski, J. Majewski and S. Satija, Biophys. J., 2004, 86, 1763-1776

31 I. Burgess, V. Zamlynny, G. Szymanski, A. L. Schwan, R. J. Faragher, J. Lipkowski, J. Majewski and S. Satija, J. Electroanal. Chem., 2003, 550, 187-199.

32 J. Cooper, R. Cubitt, R. M. Dalgliesh, N. Gadegaard, A. Glidle, A. R. Hillman, R. J. Mortimer, K. S. Ryder and E. L. Smith, J. Am. Chem. Soc., 2004, 126(47), 15362.

33 R. M. Sapstead, R. M. Dalgliesh, V. C. Ferreira, K. S. Ryder, E. L. Smith, N.-J. Steinke and A. R. Hillman, J. Amer. Chem. Soc., 2017 (submitted).

34 A. Nelson, J. App. Crystallography., 2006, 39, 273-276 (MotoFit).

35 A. V. Hughes, 2013. RasCal. Sourceforge. Downloaded from: http://sourceforge.net/projects/rscl/. (2016)

36 B. Efron, "Bootstrap methods: Another look at the jackknife.", Annals of Statistics 1979, 7, $1-26$.

37 A. P. Abbott, M. Azam, G. Frisch, J. Hartley and K. S. Ryder, Phys. Chem. Chem. Phys., 2013, 15(40), 17314.

38 A.P. Abbott, J.C. Barron, G. Frisch, S. Gurman, K.S. Ryder and A.F. Silva, Phys. Chem. Chem. Phys., 2011, 13, 10224. 